\title{
Citizens, Madmen and Children: Equality, Uncertainty, Freedom and the Definition of State.
}

\author{
Jesse Hoey \\ Cheriton School of Computer Science \\ University of Waterloo \\ Waterloo, Ontario, \\ Canada, N2L3G1 \\ jhoey@cs.uwaterloo.ca
}

January 14, 2021

\begin{abstract}
In this paper, I argue that a fundamental tradeoff between freedom and equality in human social organization is, in fact, a learnable schedule for the management of uncertainty for an individual agent operating in a social world pervaded with randomness. This implies that political organization and state structures may be viewed as different ways of handling uncertainty, each trading off freedom and equality in a slightly different manner, but each with an equivalent, and theoretically symmetric, balance between the two. I will argue that uncertainty in an intelligent agent arises from one of three primary sources. First, the agent's logical, objective, mental model of the environment may be noisy. Second, subjective, affective appraisals, of other individuals, their behaviours, and their intentions may present substantial randomness. Finally, the relationship between the objective, real, external world, to the subjective, internal world of the agent may be blurry. As I will show, in any basic two-level Bayesian model of reasoning, these same three sources of uncertainty naturally arise as three learnable parameters. These three parameters govern the operation of the model, but must be traded-off against each other. Furthermore, I will give these three dimensions of uncertainty some theoretical validity, by showing how they pervasively occur across a wide swatch of literatures as three dimensions of political freedom and equality. The relationship between the parameters and the freedoms is a key step in gaining a better understanding of political movements. For example, I discuss how the transition from mechanical solidarity to organic solidarity during the industrial revolution in the $19^{\text {th }}$ century, as described by Durkheim (2014/1893), is in fact a shift in the management of uncertainty to one focused on immediate meanings to one focused on trust. Further, such shifts may be purposefully initiated by politically interested groups, by spreading new narratives or introducing new policy. Viewed as uncertainty management, any shifts so induced should be carefully evaluated in terms of what freedoms it compromises, with smoother transitions assured for shifts that remain in a position of equilibrium with respect to freedom and equality.
\end{abstract}




\section{Introduction}

I will start with some basics about freedom and equality. These concepts are heavily discussed in the literature, of which I will barely skim the surface. My primary objective with this paper is to show that the different kinds of freedoms enjoyed by people are related in a non-trivial way to some very basic information theoretic principles about the management of uncertainty. Uncertainty, or how unpredictable the future, present or past is, can be handled by different functional levels in the brain. As a nearly trivial example, close your eyes and ears and consider how uncertain you are about what is happening around you. Opening your eyes reduces the uncertainty. However, it can also be handled higher in the brain's functional hierarchy. For example, you may see evidence that contradicts some fundamental belief you have. You may choose to disregard this evidence to preserve your certainty about the world. I will start by taking a very "zoomed out" view of politics, then move to a discussion of uncertainty and introducing some key information theoretic concepts. Then, I will discuss some key aspects of freedom and equality from sociological and political science viewpoints. I will then go more deeply into uncertainty in a hierarchical model. An appendix contains some key derivations of the information theoretic components. A longer version of this paper is (Hoey, 2020).

Politics, from the laws themselves to the people who make them, enforce them, and evaluate them, are based on some degree of balance between freedom and equality. As Rousseau (1762, p.96) points out, "every system of law [has] two main objects, freedom and equality". However, there are many ways to balance these two elements. For example, conservatives value everyone's freedom to act (e.g. to carry a gun), while liberals value everyone's equality of action. One can easily see that one cannot be free and be equal in a society of others. If everyone is free, then everyone must be unequal. If everyone is equal, then no one is free. "The liberty of some must depend on the restraint of others" (Berlin, 2002, p.171). Therefore, I can write:

$$
\text { freedom }=\frac{1}{\text { equality }}
$$

You, the reader, may feel threatened by the simplicity of this equation. However, as I will argue in this paper, freedom is nothing but an estimate of the dispersion or concentration of posterior belief distributions in a hierarchical model. Different types of freedom express themselves at different levels. Such dispersions are termed variance, and the inverse (reciprocal) of variance is precision, and very precise distributions require people to be less diverse, more similar, more equal. Thus, the equation simply states that freedom and equality are measures of the same thing, in opposite directions.

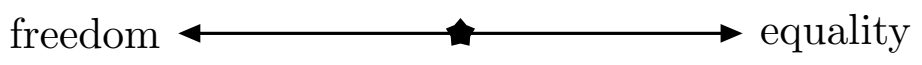

Figure 1: Freedom-Equality axis showing free energy minimum.

I can therefore represent freedom and equality on a single axis, as shown in Figure 1. On this figure, a society could be set up anywhere along the line between freedom and equality. However, using free energy principles, I can show that there is a "sweet spot" at which the group functions most efficiently. This sweet spot, shown in Figure 1 with a $\star$, is a minimum of free energy for the group, and is defined by how uncertainty is managed in a group of agents. I will return to 
this in Section 2. However, we can proceed by simply remembering that there is a spot between freedom and equality at which a social group will naturally tend to settle, and this spot will be the group's natural equilibrium on the freedom-equality axis. Another way to say this is that any group attempting to settle away from this sweet spot, will be beset by disagreement, or lack motivation, as I will show in Section 2. They will therefore be less efficient, and will tend to be dominated by groups who are at their sweet spot. Learning where an agent should situate its own, internal model of the world is something non-self-interested that an agent does, but it is something that benefits the group as a whole, and thus qualifies as a potential true form of group selection (Haidt, 2012).

In order to make this more concrete, I need to introduce some additional dimensions that will characterise freedom and equality in a way that will enable further analysis.

\section{Uncertainty}

The axis of freedom and equality actually corresponds to a fundamental trade-off in uncertainty.

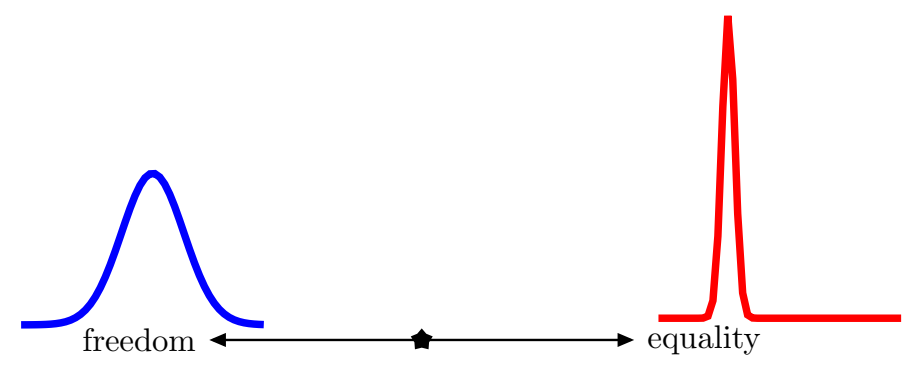

Figure 2: Diversity at freedom and equality poles.

Consider the diversity of a population. We can represent diversity as a distribution over a range of human attributes as shown in Figure 2 above the word "freedom". The wider this distribution, the more diverse the population. The one shown on the left in blue in Figure 2 has people spread across a wide range of attributes: it is very diverse, and has a lot of freedom 1 The distribution over the word "equality", on the other hand, is much less diverse, so people are spread across a smaller number of attributes, everybody is very much the same, and there is much more equality.

The variance in the group has to translate into variance in each agent's mind because of the good regulator theorem: every operational system has to be a model of its environment (Conant and Ashby, 1970). So, those operating in the society of diversity are more often going to run into diverse views of things, and therefore they will learn a more uncertain or "spread out" view of their society. They will therefore be more free to choose their own actions as there will be less constraint from the group level (as it is more spread out). Agents that live in the homogeneous society are going to have very precise distributions over the other agents in their group since

\footnotetext{
${ }^{1} \mathrm{I}$ am deliberately mixing up the colors and directions left/right here, as will become apparent later on. I am doing this because the concepts of left/right and blue/red are not relevant once they are seen simply as reflections of uncertainty management in Section 4 .
} 
everyone is similar. Actions are constrained, but equality and security are guaranteed. Security is guaranteed because, if everyone is exactly the same as you, then you can be very certain about things, you are in a state of pure equality, and you get pure security as a reward: you can predict exactly what is going to happen next. If everyone's very different then you will be very uncertain about how people will act but you can be free to act in any way you want because it won't stand out and people will know how to handle it.

Therefore, we have found two ways of doing things: precise and homogeneous, or uncertain and diverse. These methods correspond with our common notion of equality and freedom, respectively. Clearly we can't have both, and each has its advantages. Being very certain about things is good because it allows decisions to be made. An agent's certainty in something needs to be raised above a threshold for action. Being uncertain about things is also good because it allows an agent to be flexible towards changing situations and new and different people. However, these extreme values are difficult to sustain a social order over. The reason is that, in a state of perfect freedom, no cooperation is possible: there's just too much diversity. Similarly, a state of perfect equality won't succeed because everyone has to be identical, so that the system becomes very brittle to intrusions or exogenous changes.

In fact, there is a sweet spot, shown with a star in Figure 2, that trades these two off optimally in the sense that each agent is able to accurately model the group and the group is able to accurately model each agent. At this sweet spot, agent and group share a model and are best able to predict the future world.

This sweet spot in fact is the configuration of both agent and group such that the free energy (Friston, 2010) of the group is minimised, and it arises from an agent leveraging the second law of thermodynamics for its own benefit by amassing orderly states (information) at the expense of externalities (Jeffery et al. 2019). In some sense, the group has transferred as much energy as possible into maintaining a state of low entropy, a state of order, and not succumbing to chaos. The group and the individual are aligned in this case, and the heightened collective consciousness, regardless of how it is implemented, allows individuals to be more free to think, be and do (Patterson, 2014). I will return to this uncertainty trade-off when I discuss the BayesAct model. However, I can plot the free energy of the entire system vs. equality, and I find there is a sweet spot at which the system trades the two off optimally in a theoretical sense. This is the spot at which the group as a whole best ensures its survival.

In this context, one important component of the free energy is the alignment between the individual and the group. This can be broken down into two terms, one that computes the extent to which the group would have to change to fit the individual, and the other that computes the extent to which the individual would have to change to fit the group. As a function of the social uncertainty of the individual, the first term is smaller if the individual is more loosely defined, as it means the group has to shift less. The second term is smaller if the individual is more precisely defined, as it gives the individual the ability to "find" the group. Smaller free energy configurations are better because they ensure there is less "surprise" for the group.

Essentially it is easier for the group to "find" the individual (blue line in Figure 3) if he or she is loosely defined, or "flexible", and it is easier for the individual to assimilate into the group (red line in Figure 3) if they are more precisely defined (assuming they are close to equilibrium). If they are far from equilibrium, then a wider distribution will be called for as well. If we also take 


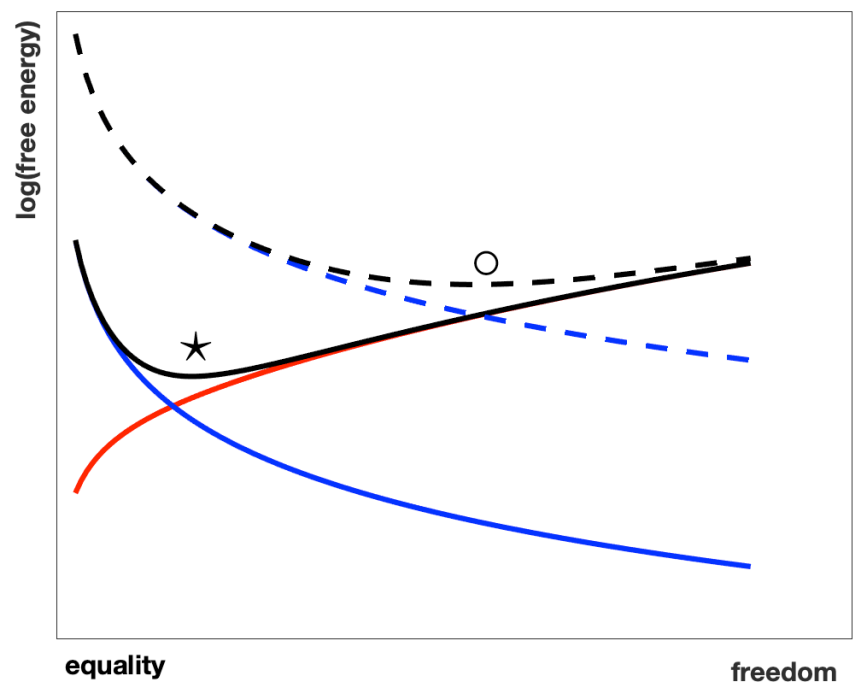

Figure 3: Free energy as a function of freedom/equality. I plot the $\log$ (free energy) for clarity only. Lower free energy is a preferable situation, but the energy created by the group trying to match the individual (blue line) is balanced by that created by the individual trying to match the group (red line) to give an overall free energy which has a "sweet spot" (minimum) at $\star$. The black line shows the free energy if these are traded off equally. The dashed lines show the situation in which the group is much less flexible because it is larger. Then, the "group" component of the free energy (individual stays fixed, group attempts to match, blue dashed line) is much higher, plays a bigger role in the resulting free energy (black dashed line), and shifts the "sweet spot" outwards towards more freedom at $\circ$.

into account the group density $2^{2}$ then the group component becomes dominant and larger, it being harder for the whole group to shift towards the agent (blue dashed line in Figure 3), and the resulting free energy has a sweet spot that has shifted upwards, towards more freedom, shown in Figure 3 with $\circ$. Such a shift may also be caused by the intensity of the relationship to the group. Those relations formed in primary socialization, for example, may have much more intensity, and therefore a much bigger effect, than those formed in secondary socialization (Berger and Luckmann, 1967, p.152). Other components of the free energy are discussed in Appendix A, which also contains a derivation of the curves shown in Figure 3 .

I will present this matching problem using an allegory of two boys searching for each other in the dark with a flashlights, as shown in Figure 4. The flashlights have an adjustable beam width, from narrow and far to wide and short. The boys get rewarded for how much light the other records (the density of light falling on him). One can see that for certain settings of flashlight beams, the boys have no hope. If one sets his beam on small and far, but the other does as well, they will have trouble finding each other. If both beams are wide, they can easily find each other, but the density of both together is low. Thus, they can either both use medium sized

\footnotetext{
${ }^{2}$ Density is similar to Durkheim's volume of collective consciousness, and relates to the effective size of the group an agent interacts with. A much denser group involves many more interactions with many members of the group, and thus carries a greater weight(Berger and Luckmann, 1967, p.152).
} 


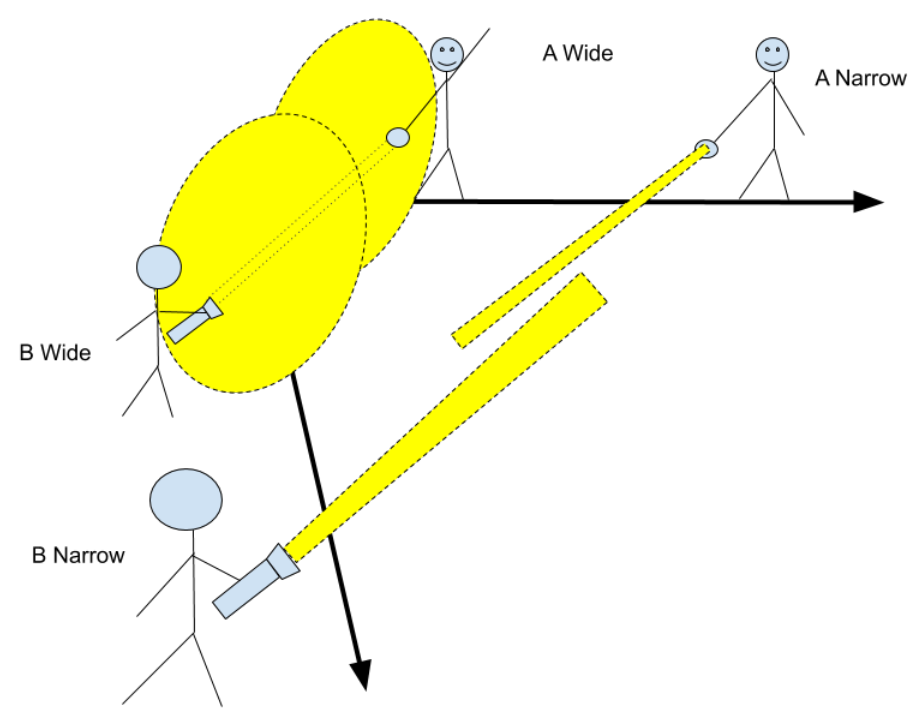

Figure 4: Allegorical example to demonstrate the uncertainty matching principle. Boy A (he can be A-narrow or A-wide) and boy B (B-narrow or B-wide) attempt to find each other's flashlights and are rewarded by the density of light falling on the other boy's flashlight.

beams, or one can use a small beam and the other a large one.

In the flashlight allegory above, consider the targets for each boy (the other boy) are like the social world, and the flashlight is the boy's predictions of how the social world will behave on a level of "meaning." The size of the target is the diversity of the social world, and represents the variance in expected behaviours. The size of the flashlight is the strength of the abstract social model the agent is building (his prior model). Therefore, the "allowable" settings are those that combine high diversity with strong abstract predictions and those that combine low diversity with weak abstract predictions. Both of these settings may work well in a network of agents with the same settings $3^{3}$ but may conflict when put in groups with different settings, as the new agent's existing model will necessarily break down (and not match). The process of learning the new "fit" will be one that may be individual dependent. Such "spotlight" metaphors have been deeply explored in the context of psychological (usually visual) attention (Crick, 1984).

\section{Mechanical and organic solidarity}

In this section, I address the question of how to move a society from freedom to equality (or the other way). Suppose we are in a situation where everyone is free, and we want to move to a situation where all are equal. We have to introduce some dependence between all these free agents and this means introducing some form of what's called solidarity. "Ideologies generate

\footnotetext{
${ }^{3}$ Note that this does not mean necessarily that the agents are homogeneous. While their parameter settings may be the same, the parameter settings define the space of possible models and agent can take on, and therefore the parameter settings are more of a measure of a social group's expansiveness. Granted, boys with wider flashlight beams will have settings that rely less on abstract meanings.
} 
solidarity" (Berger and Luckmann, 1967, p.124), and thus we can distinguish at least two primary kinds of solidarity, called organic and mechanical (Durkheim, 2014/1893). These are also known as relational and transactional forms of commitment, respectively, in (Lawler et al., 2009).

In organic solidarity, we allow everyone in the social system to be diverse, but we require that everybody has to trust one another. So everybody can be very different but you have to trust that your neighbor is going to do the right thing. There is a role-specific distribution of knowledge, all institutions are differentiated, and a division of labour is possible (Berger and Luckmann, 1967). In mechanical solidarity, on the other hand, everyone is equal and everybody follows a single plan, for example a rational plan 4 No knowledge is role-specific, and all social actions are institutional (Berger and Luckmann, 1967). Mechanical solidarity is the dimension of homogeneity.

Francis Fukuyama has written extensively about the concept of trust (Fukuyama, 1995), which he relates to Durkheim's organic solidarity, and which he defines as the "expectation that arises within a community of regular, honest, and cooperative behaviour, based on commonly shared norms, on the part of other members of that community."(Fukuyama, 1995, p.26). Fukuyama's "norms" in this quote are later clarified as "arational social habits" (Fukuyama, 1995, p.41), which is more akin to non-rational heuristics. Much like Lawler's relational commitments, trust can replace instrumental commitments (contracts) and make the economic process more efficient, and can "enforce" the solution of public goods social dilemmas like the free-rider problem (Ostrom, 2015). Further, Fukuyama explicitly points out that the "particular character of those values is less important than the fact that they are shared."(Fukuyama, 1995, p.153) That is, a large group of humans can set up a system of values that allow trusting interactions, but exactly what those values are is not as important as the trust they engender.

Now the two solidarities may be orthogonal (I have not drawn them that way in Figure 5), but are definitely dependent in any agent or group. That is, you can't have both of at the same time, although one can be changed independently of the other. The simplest way to see this is by considering differences in diversity. As diversity increases, mechanical solidarity is forced to decrease, and organic solidarity is therefore forced to increase in order to maintain the same overall degree of solidarity. Therefore, the two are traded off and must lie along a line that crosses the freedom-equality axis at the sweet spot (shown dashed in Figure 5). In fact anywhere along the line between these two axes is going to be a permissible social order with the same freedom-equality trade-off, the same sweet spot. We will see in the next section that this line is in fact a part of a three-dimensional surface

\section{Three Freedoms}

I have focused so far on connections between group members, but remember that the group is embedded in an environment which may contain many uncontrollable factors. These uncontrollable factors may be encountered by individuals, or by groups, and may consist of the actions

\footnotetext{
${ }^{4}$ Rationality is defined in this paper as decision theoretic rationality: taking the action that results, in expectation, in the highest payoff according to some cost function, see Murphy (2021) for a recent treatment of Bayesian decision theory. Perhaps a simpler way to define irrationality is as the cost of performing a certain action, where I measure cost as the risk an individual is taking by doing this action, relative to the risk he would face if he did another action that is more according to the group's prescription (social learning). That is, how much is an individual "sticking his neck out" by going with the group.
} 


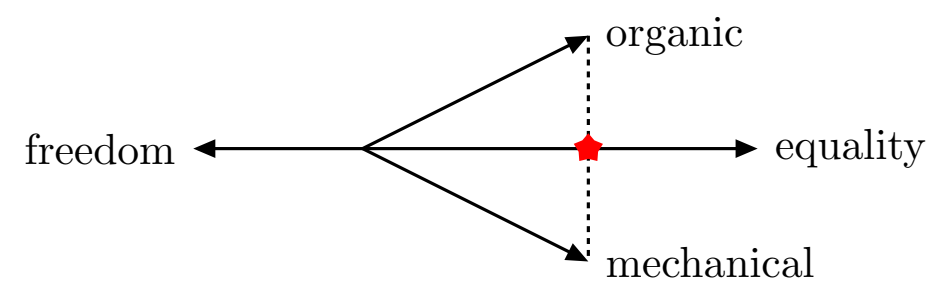

Figure 5: Freedom-Equality axis showing two solidarity axes and minimum free energy line (dashed). Mechanical and organic solidarity may be orthogonal, as in Figure 6

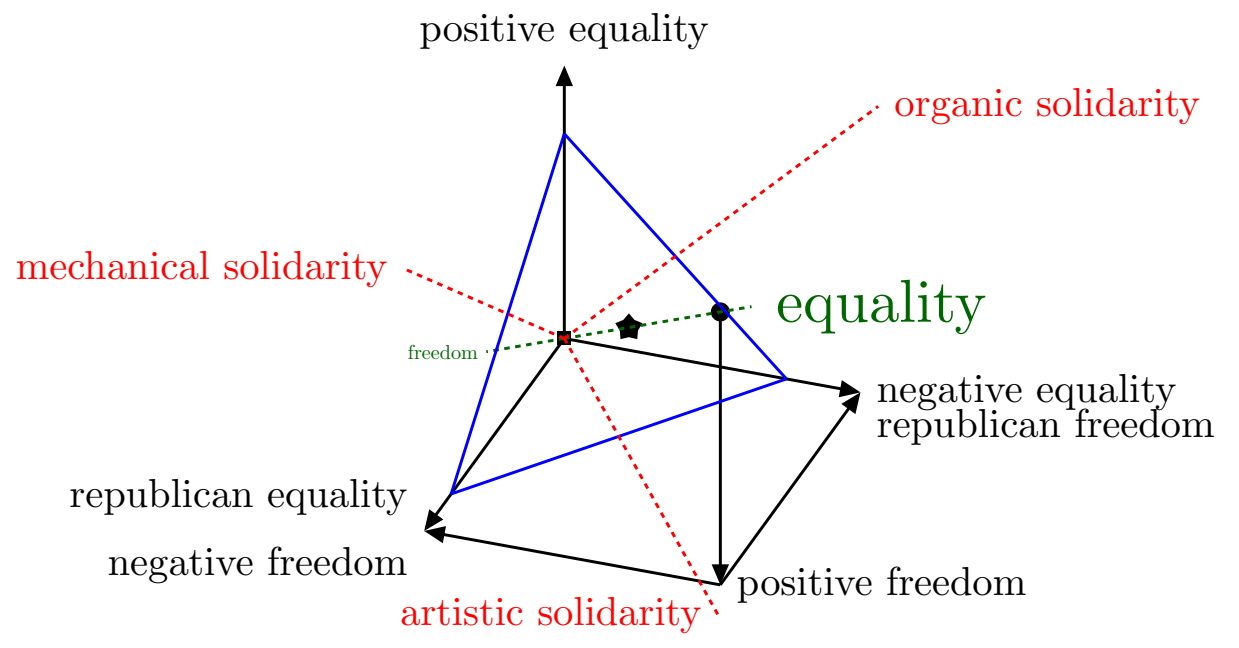

Figure 6: Simplex on the three dimensions of freedom and equality. Also shown are poles of perfect freedom and perfect equality $\bullet$, The star is in the most central position possible for a social contract. Mechanical and organic solidarity are shown, along with a third type of solidarity, artistic (see text).

of other humans in a different group (the "out-group"). How must agents deal with the uncertainty present in these uncontrollable factors? In fact, it is not the uncertainty that is the agent's concern, it is the ambiguity. Think of uncertainty as an estimate of how likely some outcome is to occur: this is the risk. Risk is an important concept, because if one can define risk, then one can make a decision-theoretically optimal decision about behaviours that lead to this risky outcome $5^{5}$ That is, an agent can rationally decide whether or not to do something, and be right about it, only when the risk is something she can estimate. However, if she cannot estimate the risk (perhaps she has never tried the behaviour so has no statistics to learn from about the likelihood of the outcomes), then her estimate of risk itself is uncertain, and we label this type of "meta-" uncertainty as "ambiguity." Ambiguity should be kept as a separate term to uncertainty (which is the entropy of $P$ ). The reason ambiguity is important as a separate concept, is that it is a factor determining when people rely more on social than individual learning, alongside problem difficulty and learning cost (Heinrich, 2016, p.64).

There are two main reasons why would an agent no longer be able to estimate risk properly. The

\footnotetext{
${ }^{5}$ Exploiting this optimality is what insurance sellers do.
} 
first is there may be some unknown (to the agent) factors that influence the outcome. These factors might be discovered should the agent try to behaviour, which it cannot do reliably without an estimate of risk. The second is the agent may lose the capacity to model an environment that has become too complex ${ }^{6}$ In either case, I will call the resulting environment invalid (Kahneman and Klein, 2009), which is synonymous with ambiguous.

I now project the freedom-equality dimension from Figure 5 into a three-dimensional space, so that it appears as a dashed green line in Figure 6. Mechanical and organic solidarity are also shown (dashed red), but as I will discuss in Section 5, they can be projected on an axis of illiberality and one of economic left/right on the simplex (see Figure 7). Solidarity increases as you move from freedom to equality. Finally, a new type of solidarity is postulated, artistic solidarity, that runs from the origin and describes any social system that is disconnected from reality. Such a social system achieves solidarity solely through emotional meanings, but otherwise is anarchical. I will not discuss this further here, but I do note that artistic freedom is one dimension of experience that is normally experienced early in life, as differentiation in the mind on these subjective dimensions may be expressed as a fantasy in the real world, like a dream disclosed (Arnheim, 1954). Further, artistic solidarity may only be possible in a situation of economic surplus, which allow for some individuals freedom from objective challenges of subsistence (Berger and Luckmann, 1967, p.81).I will not discuss this further here, as I want too focus on the three dimensional projection onto the axes of freedom you can see in Figure6 6

The slightly rotated space defined by these three freedom axes, shown in Figure 6, is both more convenient to talk about but also more directly connected to the Bayesian model that I discuss in Section 6. Before I talk about these freedoms, I point out that regardless of how we talk about this space we have to end up on (or near to) this two-dimensional surface shown outlined in blue in Figure 6. This surface is a simplex, and represents the "sweet spot" of free energy ${ }^{7}$ As with the single dimensional (linear) version in Section 3, there are numerous different ways of achieving the same freedom-equality trade-off. The trade-off shown in Figure 3 happens along all three freedom axes, meaning there is a true, ideal "sweet spot", but that there are many "nearly-sweet" spots and local minima in which a society can easily become "trapped."

Why is the space three-dimensional? Because there are three things at play: the individual, the group, and the connection between the individual and the group. Another way of saying this is the objective (the group) the subjective (the self) and the connective (membership in the group). Moral psychologists also refer to these as moral ethics of autonomy, community and divinity (Haidt, 2012). The representations in an agent's brain or mind of the social context pervades reason and thought, and the way in which each agent in each context trades off the social and individual contexts will be defined by, and will define, the social order and thus reality: "the relationship between the individual and the objective social world is like an ongoing balancing act" (Berger and Luckmann, 1967, p.134).

Let us consider now what these three freedoms mean (Anderson, 2017). Freedoms increase as you

\footnotetext{
${ }^{6}$ There is a third reason risk may be hard to estimate, which is essentially the same as the first, and so is relegated to a footnote. This third reason is that the cost of a behaviour may be too high. This basically implies the agent cannot do the action, and so leaves the outcomes unknown as in the first reason for ambiguity. These three reasons are both known to be important in gauging if an agent will favor social learning (learning by imitating others, for example) over individual learning (e.g. learning by evaluating outcomes decision theoretically) (Heinrich 2016, p.64).

TIt is not actually a plane, but rather a spherical shape or bowl shape, see Appendix A, but this planar approximation sweeps arbitrary scaling under the rug and gives us a useful analytical tool.
} 
move down each axis towards the freedom pole at the origin ( $\square$ ) in Figure6. Republican freedom means you are not subject to anyone's unaccountable will, and is also known as independence. So as you move out along the republican equality axis, then everyone becomes equivalent and dependent. Normally this is done by making all dependent on a sovereign or a monarch, such that all independence is removed by subjugation to the monarch's unaccountable will. However, a smart and honest monarch gives his subjects lots of opportunities (positive freedoms) and lets them have free choices (negative freedoms) but can intervene at any time to impose an arbitrary will to ensure everyone is steering in the same direction.

Positive freedom implies opportunity. As your group moves down on this axis, you get more and more opportunity. This happens because constraints are slackened at the group level, meaning uncertainty must be managed at levels lower down (individual) and higher up (at the corporate or government level). As you move up this axis, then opportunity becomes more constrained. Positive equality is a place where everyone is exactly acting in the same way and the world is predictable and valid (Kahneman and Klein, 2009). Therefore if you could maximise positive equality, then everyone would act according to a single plan. One such plan could be a rational plan. By defining what is good and what is bad, a rational decision maker can be used to set policy. The definition of what is good and what is bad is still needed, and as I will discuss further in Section 5, may be used by a despot for personal (perhaps only status-based) gain. It is fairly well accepted now that in invalid environment (those that present ambiguity or cognitive difficulty), agents rely more on social learning (Heinrich, 2016, p.64), which means they rely more on others, which decreases their negative and republican freedoms.

Negative freedom is defined by the freedom to choose your own actions, from whatever choices you are given. So moving towards negative equality means removing people's abilities to choose their own actions. One way to do this is by defining affective identities, and then making more stringent requirements on how actions should be coherent with these identities. These culturally approved dynamics become institutionalised, and they remove negative freedom of individuals to act in whatever way their will directs them. Thus, an increase in emotional coherence between (seemingly self-imposed) actions and behaviours, in an emotionally stratified society, leads to a reduction in the space of actions under consideration, accompanied by a corresponding increase in negative equality in which actions are constrained by social prescriptions. A state of "world closedness," extracted from a state of "world openness," is a result (Berger and Luckmann, 1967. p.51). The classic imposition on negative freedom is private property. So I can wall off a piece of ground for myself, and I have increased my negative freedom on my property 8 However, I have decreased the negative freedom of 7 billion people (well, realistically probably only a few hundred co-citizens of my rural town), and therefore overall have increased the negative equality.

\section{$5 \quad$ Political Beliefs}

Political parties, in their discourses, trade-off between the three freedoms and equalities. That is, they promise to reduce/increase taxes, reduce/increase services, or reduce/increase rules or law enforcement. The different parties represent groups of people who see the world in the same way: they use the same freedom/equality tradeoffs. This means political parties can be located on the simplex by analyzing their policies in terms of the three freedom/equality axes.

\footnotetext{
${ }^{8}$ I still require the law, and an enforcement component of government to ensure this freedom is upheld.
} 


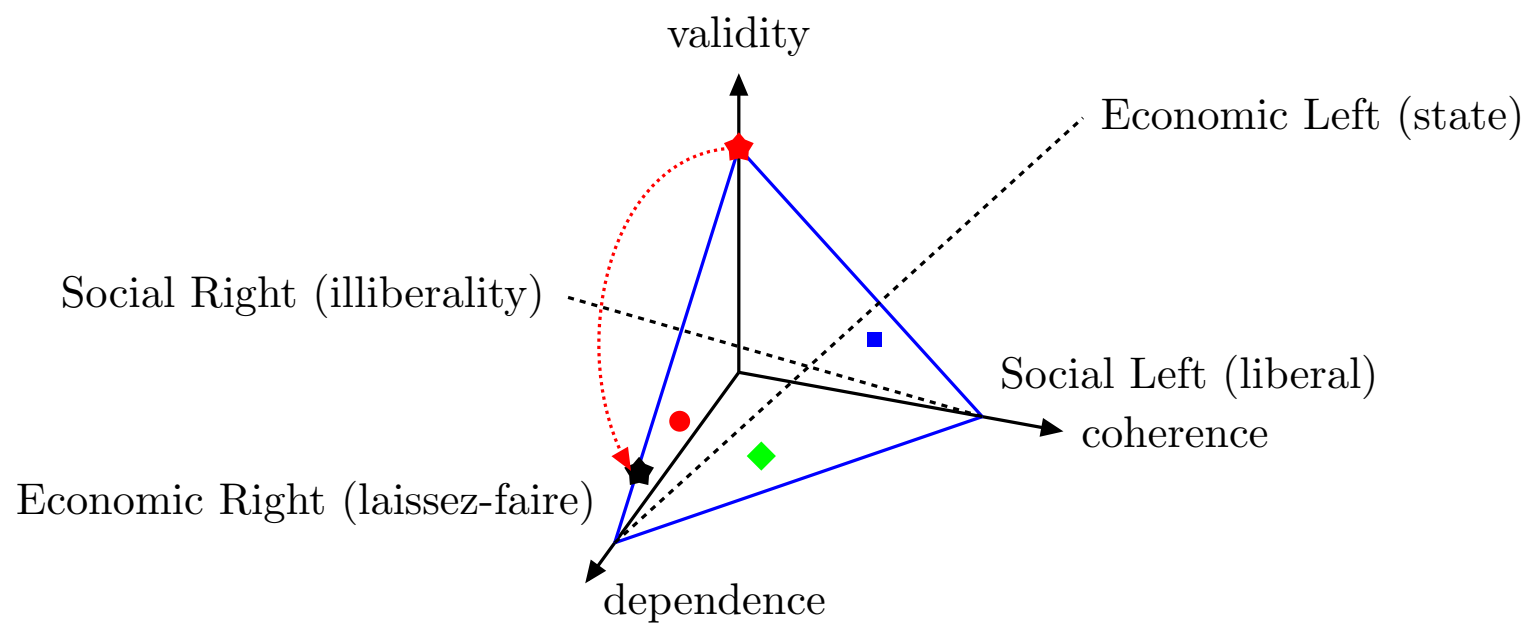

Figure 7: Dimensions of social and economic political beliefs shown as dashed lines on the simplex as projections of mechanical and organic solidarity, respectively, from Figure 5. Also shown major political parties $\bullet=$ Republican USA; $\boldsymbol{\square}=$ Democrat USA; $=$ libertarian USA; $\star=$ communist; $\star=$ Despotic; and a key shift from communist to despotic rule.

Let's look at a few extreme examples of political systems off the simplex first. In the far back corner in Figure 7 (point labeled "freedom" and $\square$ in Figure 6) are true anarchists, whose state cannot exist because nothing would ever get done, or everyone would kill each other. In the top front corner (not shown explicitly in Figure 7, at the point labeled "equality" in Figure 6) are true communists, whose state can also not exist, because of mass confusion and disagreement about the definition of state. On the simplex lie workable social systems. Modern American republicans, who are illiberal and economically conservative, would lie in a position of high mechanical solidarity, and low organic solidarity, or one of high republican equality, coupled with high negative freedom. They are shown as a red circle ( $\bullet$ in Figure 7). Modern American democrats, and most Canadian parties, are somewhat near the center, but perhaps a bit higher on the organic solidarity and a bit lower on mechanical solidarity, or a bit more negative equality, a bit more republican freedom ( $\mathbf{\square}$ in Figure 7 ).

Highly rationalistic states (with no positive freedom) were promoted throughout the 18th and 19th centuries (Kant, 1785, Marx, 1867), but are vulnerable to exploitation by despots, a feat accomplished in the 20th century to the detriment of a great number of people. Such despotic autocracies replace rationality with divinity, and strip citizens of republican equality, replacing it with positive freedom. This shift towards the economic right (less government) by a socially right wing, yet economically left wing communist party ( $\star$ in Figure 7 ) is possible with a despotic leader who, by re-defining what is meant by "rational" or "normal", and by marginalizing or dehumanizing certain sub-groups, moves the group towards the dependence corner $\left(\star\right.$ in Figure 7 ) $9^{9}$

\footnotetext{
${ }^{9}$ The despot does this using a bait-and-switch trick. He (used with gender prejudice) first convinces the people that a rational way of doing things will bring everyone unlimited positive and republican freedom. He further convinces the people that he perfectly embodies this rational way (perhaps by divine right) and that he is perfectly aligned with the people's ideology. He may easily do this by engaging with his targets in "theoretical squabbles that are quite irrelevant to their interests" (Berger and Luckmann, 1967, p.125) (e.g. abortion or gay marriage). Once he has the buy-in from the people (the alignment of their moral matrices with his, and the acceptance of
} 
These marginalised groups, labeled as non-human "barbarians" are revealed to be so because they are denying their own nature (which is, in fact, unbeknownst to them, the newly defined "normal"). Liberal political philosophy contains the same loophole, in fact, as everyone is free to act as they please, but republican freedom is only guaranteed by some democratic decisionmaking process that must exclude people incapable of making an informed choice in a certain way, e.g. "madmen and children" (Friedman, 1962). That is, the point at which decisions are made by a government attempting to balance a liberal view with other, more centrist views, must exclude those not able to tell the difference, but leaves open how to define "madman", and has the same vulnerability to despotism (e.g. the despot can label some group as "madmen" or "savages" or "animals" in order to exclude them from decisions). It may be possible to avoid this using an automated method of finding a neutral decision making point that is independent of any political party.

Libertarians are big on positive and negative freedom, and are willing to sacrifice some republican freedom to get it. Being anti-government and secular, they require some kind of authority, but are happy to hand this over to big corporations (aka private governments (Anderson, 2017)) . They are shown as a $\diamond$ in Figure 7. The final US group of "progressives" (also labeled "woke left") are those who place enormous significance on negative equality, ensuring the rights of all are guaranteed. They are fine with a heavy state, but are libertarian-like in their refusal to adhere to rationalistic views. They thus fall down in the far corner labeled "coherence" in Figure 7. Most social groups lie somewhere in the middle region of this space.

Movement across the simplex is possible, and may be caused by exogenous changes like natural disasters, or willful political, social or economic change, as in the despot example above. Another example is Durkheim's study of the shift from mechanical to organic solidarity during the industrial revolution caused by social and economic changes. We can also consider the shifts caused by the events of 2020, as freedoms were massively changed very suddenly. Societies may react to these shifts by reverting to their group identities, likely causing increasing political polarization and other negative effects. Careful considerations of the multi-faceted problem of freedom and equality may be helpful for social progress.

Datasets of impression variances are lacking, but datasets of variances in sentiments, which in BayesAct are a measure of dependence, is sufficient to give one of the three axes. Data collected in the summer of 2020 asked 384 unique participants to rate 85 occupations (identities like "grocery clerk") on E,P,A, giving 192 responses per concept rated (see (Quinn et al., 2021) for full details of the study). The number of raters per stimulus (concept rated) is above the number usually used in ACT sentiment studies to achieve reliabilities above 0.90 (Heise, 2010). Respondents to the survey were U.S. citizens drawn from Qualtrics panels. A quota sampling method was used to match the sample of respondents who completed each survey module to the population proportions reported by the 2010 Census in its categorical measures for age, race and ethnicity, level of education, and gender. The survey also included the GSS political belief question (not controlled for), which asks participants to rate their political orientation as Independent, or as Strong, not very strong, and "close to" both Republican and Democrat. I

him as ideological leader), he starts to move his own policies until he has removed sufficient republican freedom from the people, gaining enormous resources in the process. This is documented through history (Orwell, 1945). In fact, this trick can be used from anywhere on the illiberal side of the simplex in Figure 7 , as groups in this region require a divine leader who can define things as he pleases. On what I have labeled the liberal side, this is more difficult, as individual identity takes precedence over divine meaning. 


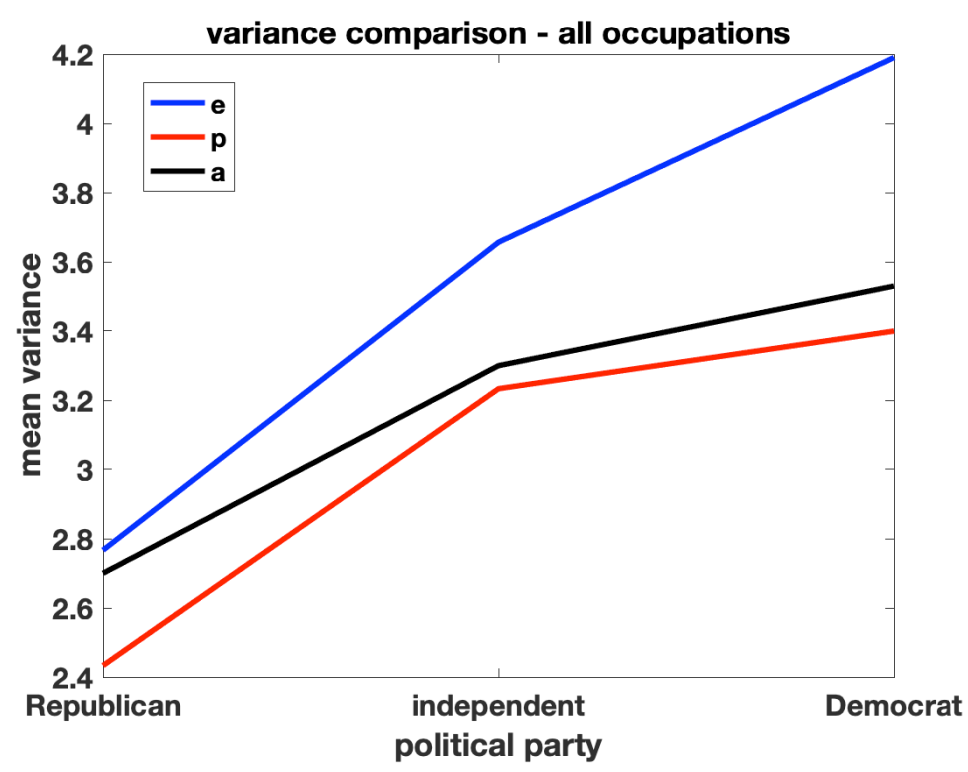

Figure 8: Data showing changes in variance in sentiments as a function of political orientation. One can see a clear trend that correlates variance with left-leaning political beliefs.

discard all participants who did not answer the political belief question (there was only one), and group all democrats together, and all republicans together, yielding a 3-way comparison. This study categorised occupations into "essential" and "non-essential" (as defined by pandemic lockdown rules that were in place when the study was run). There were 44 non-essential and 41 essential occupations, with 71/95 ratings per concept for democrats (non-essential/essential), $55 / 46$ for independents and 65/50 for republicans. Respondents who failed attention checks were not included in the analyses or the Ns reported above.

Unbalanced (non-matched and non-weighted) data give the plot shown in Figure 8. A clear trend is shown between variance and the political left, with more democratic-leaning people having more dispersed sentiments as a population. This data is in the process of being cleaned and balanced, so these numbers may change, but I compute significance in two ways, as reviewed in Appendix B.

I believe this evidence is fairly weak, as it only focussed on one dimension. However, a study is in the works to measure all three dimensions, with the dimension of validity presenting some problems that need to be overcome first.

\section{$6 \quad$ Bayesian Modeling}

The three freedoms that I've been describing are actually three different ways of managing uncertainty in a two-level Bayesian model. Why a two-level model? It's thought the brain has at least five levels of functionality (Lane, 2000), but with only two we can already observe some of the uncertainty tradeoffs that we see in real social systems. In some sense, I am abstracting the five levels into two, or I am examining one particular "adjacent" set of two levels, roughly at the level of abstraction at which the discrete symbols of language become meaningful. the 
theory I am presenting is likely to be a useful abstraction at the level of the so-called "global workspace" (Barrett and Satpute, 2013, VanRiullen and Kanai, 2021). This level at which I am abstracting is also motivated by the expectation that life is "structured by moral norms, and is designed to learn and internalize such norms" (Richerson and Boyd, 1998). Emotional and social prescriptions truly embedded in agent's minds were selected for as conducive to more efficient group behaviour (Haidt, 2012; Turner, 2016), and thus led to the emergence of persistent social structures. These social structures are encoded in a level that transcends facts and language, a level of sentiment, of feeling.

So let's imagine a two-level model that has one level for managing (representing) the objective world, the one of logic, facts, actions and objects, and has a second level for managing the affective world, the one of memory, behaviour, social relationships and trust. I make a distinction between action and behaviour in that the first describes linguistic labels (propositions) denoting actions, such as give something to, while the second describes the affective meanings of an action, say very positive and a bit powerful in this case. While the first level is connected to objective reality, for example to sensors/actuators in a robot, the second is connected to subjective reality, that is, emotional signals to/from others 10 Throughout this section, do not lose sight of the fact that all probability distributions I refer to define the relative likelihoods of state and of actions/behaviours.

Each level in this model has a certain degree of uncertainty to it ${ }^{11}$ where uncertainty is really a characterization of degree of belief, following a Bayesian view that puts the existentialism of the world into the mind itself (Pearl, 1988). Further, there must be uncertainty in the connection between the levels, which turns out to be important. That is, once we propose two different functional "levels" of processing in the brain, they must be combined in some way to produce, in the end, motor signals for purposeful action. The way this combination happens can be more or less precise, that is, the levels depend more or less on each other. I will call the three types of uncertainty denotative (objective model), connotative (subjective model), and connective (objective-subjective connection model) ${ }^{12}$

Degrees of uncertainty are intimately connected with freedom, as I discussed in Section 2, What types of freedom are associated with each of these three type of uncertainty? Denotative uncertainty is the same as positive freedom, in that invalid environments are ones in which everyone is doing different things, and so positive freedom is maximised. When all are "forced" to behave in some way (e.g. rational), then positive equality is maximised (everyone is following the same plan), but of course, positive freedom is minimised.

Connotative uncertainty is the same as negative freedom, as it releases people from social norms and prescriptions that cause constraints on their actions. Institutions increase equality along this axis, as discussed in Section 4. Finally, connective uncertainty is the same as republican freedom, as it releases people from adherence to some externally defined reference point. A

\footnotetext{
${ }^{10}$ This sounds problematic in that I am assuming an artificial split between what is sensed objectively and what is sensed subjectively. However, recent evidence shows that visual inputs go through three pathways: one for object identification, one for object location and affordance, and a third for the dynamic aspects of social perception (Pitcher and Ungerleider, 2020). Thus, a signal separation based on content at a fairly low/early level in the brain may provide a separation of observations in exactly the way I have assumed in BayesAct.

${ }^{11}$ Also called "noise", uncertainty is implemented in a deep network as a randomness in firing rates across groups of neurons (Eliasmith, 2013).

${ }^{12}$ Note there is no constraint on what the model actually is, so long as it has a use for these three types of uncertainty.
} 
great deal of connective certainty requires a leader, who, since positive and negative freedoms are maximised in this state, must be authoritarian. This leader must define what is "good" and "bad" for the group to be cooperative, since everyone within the group has so much freedom to follow their own definitions. Connotative certainty also requires a leader, but it can be defined as a social contract, since this configuration requires people to give up their negative freedoms to obtain this connotative security.

Therefore I can say that the setting of parameters of uncertainty (variances) in a two-level Bayesian model corresponds to the setting of political belief in the resulting group and the placement on a three dimensional simplex of freedoms. The primary insight we gain is that all such settings are equivalent in terms of their tradeoffs between equality and freedom in general, that is, along the dashed green axis in Figure 6, which is what we really should care about. The precise way in which this balance is achieved matters less, and conflicting mixtures of uncertainty management should be avoided. Narratives that can give justifications for actions in line with one group or the other may be important to guide marginalised groups towards fair solutions. Losses of republican freedom can be compensated for by underlining the associated gains in positive freedom, for example.

\subsection{Bayesact}

BayesAct is a two-level model of human intelligent and affective reasoning (individual and social) that explicitly represents the three dimensions of uncertainty in a simple and measurable way by leveraging the machinery of affect control theory (ACT) (Heise, 2010; Hoey et al., 2016; Schröder et al., 2016; Asghar and Hoey, 2015; Hoey and Schröder, 2015). ACT is a model of emotional coherence based in language that was founded on the control principle of Powers (1973), which states something very reminiscent of the free energy principle: that people try to minimise incongruencies by controlling their perceptions. He transposed this to the sentiment space of Osgood et al. (1957), imposed a denotative structure from symbolic interactionism (Mead, 1934), and added affective dynamics (Gollob, 1974). ACT is a computational model that has been used to predict classes of human behaviour in a variety of settings (MacKinnon and Heise, 2010). ACT maintains a deterministic and static denotative model as an actor-behaviour-object-setting state (e.g. "doctor advises patient in clinic", and an associated deterministic but dynamic connotative model: a dynamical system in Osgood's three-dimensional space of affective meaning: evaluation, potency and activity. This dynamical system represents values, or evaluative knowledge, which can be contrasted with declarative and procedural knowledge that are represented in the denotative model.

BayesAct combines these mechanics with a formal decision theoretic model, a partially observable Markov decision process, or POMDP (Åström, 1965; Boutilier et al., 1999), extensively used in operations research (Puterman, 1994). A POMDP instantiates a temporal frame or structural representation (Russell and Norvig, 2010). Frames, as schemas, are a classic structure used in early artificial intelligence (AI), Knowledge Discovery and Data Mining (KDD), and Information Retrieval (IR) research that assigns a label and interpretation to each object, fact, relation and event that constitute a particular situation. Such structures are typically logical and discretevalued to enable ease of use in a computer program. For example, we might label the positions of pieces on a chess board, or predictions about how a game will turn out given a sequence of moves, or the bids in a negotiation. The inclusion of ACT means the model must be augmented 
with labels for identities and behaviours corresponding to ACT's denotative model, but with added noise modeling. These labels can then be interpreted as distributions in a sentiment space using a measured dictionary. This sentiment space thus complements the denotative state I have been describing so far, with a connotative state (which in fact is 18 dimensional). The model is fundamentally based on the symbolic interactionist idea that symbols (language) provide order for "the subjective apprehension of biographical experience" (Berger and Luckmann, 1967, p.97). Symbols are then reified elements of exactly these same subjective apprehensions. Thus, learning and being become one single experience. The combination of symbolic and affective interpretations is what enables generalization: once the symbol "doctor" is assigned to someone, expectations for her behaviour become defined as generally as possible with respect to her occupation. That is, I expect her to do something good and powerful, but I'm open to a range of actual objective actions that could be in play in the current situation. For example, if the current situation is a court-room, I still expect her to do something good, such as testify honestly, and powerful, such as speak authoritatively.

Frames form the foundation of much knowledge representation work in AI, but have been efficiently implemented using Bayesian networks (BNs), which can be used to compute a distribution over all possible worlds modeled by a particular frame (Pearl, 1988). This probabilistic model then rests on the structural ontology and temporal logics that are proposed in the frame. Bayesian decision networks generalise the goals in frames as preference functions that rank all possible outcomes using a numeric scale, e.g., a utility function (von Neumann and Morgenstern, 1953). BayesAct complements this denotative model (the variance of which is the invalidity), with the ACT-based connotative model (the variance of which is the coherence), and a model of the relationship between them, the somatic transform (the variance of which is the the dependence) (MacKinnon and Hoey, 2020). For example, in a government policy decision, the objective facts may include the amount of money spent or saved, and long-term estimates from potentially complex predictive models, and the utility is financial. The denotative temporal dynamics may describe immediate and longer-term effects, enabled by adding more latent state, and allow for the construction of a policy that optimises over some definition of utility based on the same features. The denotative temporal dynamics may also encode norms of behaviour that indicate the normative choices to make for any given identity-behaviour combination (e.g. a "citizen" should not "free ride" on other "citizens", or a more extreme example, a "son" should not "make love to" his "mother", which incidentally is emotionally coherent, but denotatively taboo).

The connotative dynamics are ACT-based, and will encode the relative freedom tradeoffs for whatever group they are applied to. That is, for some particular configuration of the denotative state, including a definition of identities, a connotative distribution results that may be used to compute how emotionally coherent various behaviours are. This connotative coherence is one of "feeling" or "intuition", which may override any norms. I will call such coherence "prescriptive" rather than "normative." A striking example is a trolley problem, in which it is logical to throw the switch on a runaway trolley so that it kills only one person instead of five, a strong connotative prescription against "killing someone" may take over for many people and prevent this logical strategy. Another example is an ultimatum game, in which one player is given $\$ 10$, and can give any amount he wishes to the other player. While logically the proper amount to give is $\$ 0$ (or $1 c$ if the game is repeated and the other player has a choice not to play), most humans will fork over about $20 \%-40 \%$ of the amount they are given. 
BayesAct may be summarized by looking to the seven concepts or devices that describe "culture" as outlined in Small et al. (2010), and re-iterated in (Vaisey and Valentino, 2018). These seven concepts have associated concepts in the BayesAct framework as follows:

- Values correspond to the parameters for the management of uncertainty (coherence, dependence and validity), and the dynamics (changes to) these parameters as a result of interactions. This interpretation of values corresponds to evaluative knowledge (Patterson, 2014: Martin and Lembo, 2020), and so steps one level back from describing the "ends toward which behaviour is directed" (Small et al., 2010, p.14) to describing the ways in which those ends can be computed.

- Frames correspond to denotative beliefs (priors and posteriors). Frames are heavily used in artificial intelligence as, for example, statements about what conditions need to occur before a certain plan can be carried out (pre-conditions), or what the effects of actions will be (Poole and Mackworth, 2010). Frames may include goals, or more generally utility functions that encode preferences. These declarative elements of an agent's value system are those the agent seeks to optimise over, but only within the limited vision afforded by the blinders of his connotative system, of his values. In fact, it may be the case that values are reified into objective reality in any institution as preferences of particular types of agents, and thus allows bootstrapping of the utility function onto the values. However, starting with a prior set of preferences can help the agent get started in the right direction.

- Repertoires correspond to denotative policies or strategies of action, referred to in artificial intelligence as heuristics and in cultural sociology as toolkits (Lizardo and Strand, 2010).

- Narratives correspond to denotative dynamics and histories, and can be used to establish trajectories that were followed in history or that may be followed in the future. Narratives are strengthened by the denotative coherence of the story being told, and are often constructed "after the fact" as an explanation that justifies certain behaviours (Mercier and Sperber, 2017; Haidt, 2001; Berger and Luckmann, 1967). After an alternation of self (e.g. a religious conversion, a change in relationship), negative narratives about the old self, may serve to bolster support, beyond what is provided by positive narratives, for the new (Berger and Luckmann, 1967, p.160).

- Symbolic Boundaries correspond to the somatic transform, and associate morality, culture, and identity to narratives.

- Cultural Capital correspond to connotative beliefs in general, and in particular over highstatus elements of culture (so there is an ordering of capital). In BayesAct the restriction of cultural capital to only high-status elements is relaxed, and capital can be held over any connotative belief. In a criminal gang, for example, the capital may be the perceived and transmitted "bad-ass-ness" of a certain individual.

- Institutions are a somewhat broad umbrella, corresponding to both connotative and denotative "programs" in BayesAct, and so encompass all of the above in some sense. Institutions are "called up" from memory upon entering some institutional context, and imply a values setting, prior beliefs (denotative and connotative) and a set of strategies/heuristics. 
All three conceptions of institutions mentioned by Small et al. (2010, p.19) are handled in BayesAct:

- formal rules of behaviour (norms in BayesAct that are enforceable),

- norms of behaviour that are not enforceable (toolkits/heuristics)

- taken-for-granted understandings that frame (connotative beliefs)

The tradeoffs I have been discussing above are implemented in BayesAct to explain a number of cognitive biases (Hoey et al., 2020), to relate to theories of emotion (MacKinnon and Hoey, 2020), and to explain relationships to the sociology of culture (Hoey, 2020). BayesAct has the three parameters of uncertainty described above as either settable (in the case of sufficient prior information) or learnable for any specific group of people that is sufficiently large to gather statistics on.

\subsection{Learning}

This learnability is a key feature of a POMDP-based approach, as it allows to connect to work in artificial intelligence (AI), particularly in reinforcement learning (RL, a type of machine learning (Sutton and Barto, 2018)). Artificial intelligence has largely relied on decision-theoretic models of reasoning (the POMDP being the canonical and most general one), and have attempted to "simulate" altruism or influence by adjusting utility functions or priors such that altruistic or influential behaviour becomes more valuable (Fehr and Schmidt, 1999; Loewenstein and Lerner, 2003, Jaques et al., 2019). RL has recently been used with much fanfare in the solving of seemingly complex human games like Starcraft (Vinyals et al., 2019). However, when you realise that the computers could much more easily defeat humans in many games by ignoring the artificial restrictions put on it so that it plays more human-like (e.g. it has an artificial "human-like" reaction time of $100 \mathrm{~ms}$, a common addition to many game engines), then these games appear to be perhaps too simple, and this is likely because they do not involve any social contact. Attempting to create an artificial judge, for example, would be a situation requiring some degree of alignment on dimensions of morality.

In any case, RL does confront a key challenge for any learning system, and that is how to tradeoff exploitation of known knowledge with exploration of new situations. Exploitation is normally handled with a decision-theoretic reasoner that optimises the expectation of future rewards or costs. Exploration is usually relegated to something "emotional" such as "optimism under uncertainty": if you haven't tried something, assume its good. This encourages exploration, which can be modeled in a Bayesian framework as a search over model parameters, so-called Bayesian reinforcement learning (Poupart et al., 2006). In fact, if one views the connotative level in the BayesAct model as a parameterization of the model space of the denotative level, then BayesAct is, in fact, a Bayesian reinforcement learner.

The BayesAct explanation for altruism or influence is therefore quite different. It arises because of an identity "dance" that is scripted to a certain degree. One cannot dance the two-step in any way one chooses! In fact, one's negative freedom on the dance floor is severely hampered by the expectations of moves by one's partner. And it just so happens that the dance script, being agreed upon beforehand, encodes cooperative moves between partners. In BayesAct, the connotative level "illuminates" only a certain part of the denotative future that is altruistic, 
perhaps. Suppose, to carry this dancing analogy a bit further, that a set of dancers wanted more negative freedom: they didn't want to be constrained by the pattern of the dance moves anymore. Well this is simple, they simply re-write the contract, redefine what a dance move is by making it much less constrained. Now the coordination is lost, but it can be recovered by sacrificing some republican freedom, and agreeing to move according to the music beat. Then all dancers are moving again in concert, albeit using a much different overall paradigm. BayesAct has a decision theoretic reasoning component that optimises a reward function (or a prior expectation over future states), but only within the "illuminated" region defined by the values of the group. In the dance allegory, a dancer may be attempting to woo a particular partner, but can only do so within the culturally defined constraints of the dance script. I used the term "values" here to mean the connotative prescriptions, as it must be distinguished from "interests", which correspond to the denotative utility function (Martin and Lembo, 2020).

More scripted dance moves correspond to narrower flashlight beams, and are exploitative of affective norms in a social group. However, these affective norms have a positive side-effect: they encourage denotative exploration, as noted in Section 6.1. To see this, imagine Mr. Perfect (Hargreaves, 1990a) who everything always goes right for. Mr. Perfect can certainly expect that anything he has not tried will work out well, perhaps even better than anything he's experienced yet! He therefore has an optimism under uncertainty learning bias. However, he really must trust that he is, in fact, Mr. Perfect, and not, for example, Mr. Wrong (Hargreaves, 1990b), who, as you can imagine, everything always goes wrong for, which is the opposite of Mr. Perfect as far as I can tell. Less scripting of the dance moves, but following the beat, corresponds to wider flashlight beams, and more explorative of affective norms in a social group, but also more exploitative of the denotative state, as their focus is on optimising individual reward (e.g. a focus on the music). This correlates well with evidence that liberals are more exploratory, open to novelty, while conservatives are more exploitative, and desirous of tradition and leadership, and more reactive to threats (Haidt, 2012, p.325) ${ }^{13}$ BayesAct can seamlessly transition between the two modes of operation, and can serve as a translator between them by defining a neutral point that optimises between exploration and exploitation for the good of both groups.

Related views include the identification of negative affect (valence) with increased uncertainty (FeldmanHall and Shenhav, 2019) or with change in free energy (Joffily and Coricelli, 2013), or expected free energy (Hesp, 2018). One component of identity (esteem) is used to modulate a reward function in (Moutoussis et al., 2014) in order to make cooperation the more salient policy in a social dilemma. In a similar vein, Jaques et al. (2019) consider social influence as an additional factor in an agent's utility function in a sequential social dilemma, and the relative weights of this factor can be learned by an agent about other agents. Other models also combine "hot" and "cold" cognitions, such as Thagard (2006, HOTCO) and Glöckner and Betsch (2008), and apply them to moral dilemmas Simon et al. (2015). Recent work has combined ACT with HOTCO in Eliasmith's neural framework (Kajić et al., 2019). These models operate in much the same way as BayesAct, but come with a different learning bias (discriminative, so a prior is more difficult to specify), and a different mechanism for specification (as nodes with weights). Nevertheless, I suspect that many of the same conclusions would hold for these models as well.

\footnotetext{
${ }^{13}$ It does not, as far as I know, correlate with any preference for different dance moves for different political beliefs, and therefore the dance floor, as the flashlight, is destined to remain only an allegory.
} 


\section{Conclusion}

In this paper I described a fundamental connection between the modeling of uncertainty in a group of agents, and the freedoms which that group enjoys (or not). The inverse relationship between freedom and equality was also explored, and I showed how three known dimensions of freedom correspond to three types of uncertainty in a two-level Bayesian model. Such a model is implemented in BayesAct, a formal dual-process model that takes uncertainty into account explicitly (Hoey et al., 2020). I discuss how this model could be used to inform balanced political choices. In summary, knowing that we can represent things in these three dimensions of freedom allows us to gain deeper insights into how political movements are moving about in this three-dimensional space, and thus to understand, and possibly influence, the political and social change around us better. A few last points may be made before I close.

The observation that people empathise more with others who have the same "moral matrix" is evidence for the same type of alignment process of the individual to the group (Haidt, 2012). The moral matrix in this case is simply the position on the freedom-equality axes, but is much more context dependent than Moral Foundations Theory and does not consider the intuitionist component to be necessarily heritable or stable and dispositional. This is in line with recent (lack of) evidence for the five-factor moral foundations model, pointing instead to a two dimensional model (Smith et al. 2017). Instead, BayesAct considers "intuitive reasoning" to be based somewhat on a long-term history of identities that are summed up in the self, combined with a situational identity chosen by the individual given the context. That is, BayesAct models shift their "moral matrix" according to the group or social context, and both group and individual do a situational "dance" in order to find an aligned matrix to work from. The result is a clear prescription for solving problems of political polarization, and it is not political, and is reasonably well known now (Talisse, 2019). It is simply that each person must be (1) confident in who they are, self-affirmed; and (2) truly empathetic with the people on the "other side." This finding is reflected as well by (Facciani and Brashears, 2019), and the results of Bail et al. (2018) show that should (2) not hold, then polarization increases as homogeneity increases.

Finally note a close correspondence between atheists and rationalists, the pole of validity, as noted by (Haidt, 2012). This leads to a tantalizing association between monotheists and the pole of coherence, and between polytheists and the pole of dependence. Although high dependence groups may follow a monotheistic religion, they tend to usurp it quite a bit by reinterpreting scripture in whichever way is most convenient to explain their actions.

\section{References}

K. J. Åström. Optimal control of Markov decision processes with incomplete state estimation. J. Math. Anal. App., 10:174--205, 1965.

Elizabeth Anderson. Private Government. Princeton University Press, 2017.

Rudolf Arnheim. Art and Visual Perception. University of California Press, 1954.

Nabiha Asghar and Jesse Hoey. Monte-Carlo planning for socially aligned agents using Bayesian affect control theory. In Proc. Uncertainty in Artificial Intelligence (UAI), pages 72-81, 2015. 
Christopher A. Bail, Lisa P. Argyle, Taylor W. Brown, John P. Bumpus, Haohan Chen, M. B. Fallin Hunzaker, Jaemin Lee, Marcus Mann, Friedolin Merhout, and Alexander Volfovsky. Exposure to opposing views on social media can increase political polarization. Proceedings of the National Academy of Sciences, 115(37):9216-9221, 2018. ISSN 0027-8424. doi: 10.1073/pnas.1804840115. URL https://www.pnas.org/content/115/37/9216.

Lisa Feldman Barrett and Ajay Satpute. Large-scale brain networks in affective and social neuroscience: Towards an integrative functional architecture of the brain. Current Opinion in Neurobiology, 23:361-372, 2013.

Peter L. Berger and Thomas Luckmann. The Social Construction of Reality. Doubleday: Anchor Books, 1967.

Isaiah Berlin. Liberty. Oxford University Press, 2002.

Craig Boutilier, Thomas Dean, and Steve Hanks. Decision theoretic planning: Structural assumptions and computational leverage. Journal of Artifical Intelligence Research, 11:1-94, 1999.

Roger C. Conant and W. Ross Ashby. Every good regulator of a system must be a model of that system. International Journal of Systems Science, 1(2):89-97, 1970. doi: 10.1080/ 00207727008920220 . URL https://doi .org/10.1080/00207727008920220.

Francis Crick. Function of the thalamic reticular complex: the searchlight hypothesis. Proceedings of the National Academy of Sciences, 81:4568-4590, 1984.

Emile Durkheim. The Division of Labor in Society. Free Press, 2014/1893.

Chris Eliasmith. How to build a brain: A neural architecture for biological cognition. Oxford University Press, New York, NY, 2013.

Matthew Facciani and Matthew E. Brashears. Sacred alters: The effects of ego network structure on religious and political beliefs. Socius: Sociological Research for a Dynamic World, 6:1-16, 2019 .

Ernst Fehr and Klaus M. Schmidt. A theory of fairness, competition, and cooperation. The Quarterly Journal of Economics, 114(3):817-868, 1999. URL doi :10.1162/003355399556151.

Oriel FeldmanHall and Amitai Shenhav. Resolving uncertainty in a social world. Nature Human Behaviour, 3:426-435, April 2019.

Milton Friedman. Capitalism and Freedom. University of Chicago Press, 1962.

Karl Friston. The free-energy principle: A unified brain theory? Nature Reviews Neuroscience, $11((2)): 127-138,2010$.

Francis Fukuyama. Trust: The Social Virtues and the Creation of Prosperity. Free Press, 1995.

Andreas Glöckner and Tilmann Betsch. Modeling option and strategy choice with connectionist networks: Towards and integrative model of automatic and deliberate decision making. Judgment and Decision Making, 3(3):215-228, March 2008. 
Harry F. Gollob. The subject-verb-object approach to social cognition. Psychological Review, 81:286-321, 1974.

Jonathan Haidt. The emotional dog and its rational tail: A social intuitionist approach to moral judgment. Psychological Review, 108:814-834, 2001.

Jonathan Haidt. The Righteous Mind: Why good people are divided by politics and religion. Vintage, New York, 2012.

Roger Hargreaves. Mr. Perfect. Egmont, UK, 1990a.

Roger Hargreaves. Mr. Wrong. Egmont, UK, 1990b.

Joseph Heinrich. The WEIRDest people in the world. Farrar, Strauss and Giroux, New York, 2016.

David R. Heise. Surveying Cultures: Discovering Shared Conceptions and Sentiments. Wiley, 2010.

Casper Hesp. Hedging your bets: and active inference formulation of valence and arousal. Unpublished reserach project, 2018.

Jesse Hoey. Structure is the management of uncertainty in groups. under review, 2020.

Jesse Hoey and Tobias Schröder. Bayesian affect control theory of self. In Proceedings of the AAAI Conference on Artificial Intelligence, pages 529-536, 2015.

Jesse Hoey, Tobias Schröder, and Areej Alhothali. Affect control processes: Intelligent affective interaction using a partially observable Markov decision process. Artificial Intelligence, 230: 134-172, January 2016.

Jesse Hoey, Neil MacKinnon, and Tobias Schröder. Denotative and connotative control of uncertainty: A computational dual-process model. under review, 2020.

Natasha Jaques, Angeliki Lazaridou, Edward Hughes, Caglar Gulcehre, Pedro Ortega, Dj Strouse, Joel Z. Leibo, and Nando De Freitas. Social influence as intrinsic motivation for multi-agent deep reinforcement learning. In Kamalika Chaudhuri and Ruslan Salakhutdinov, editors, Proceedings of the 36th International Conference on Machine Learning, volume 97 of Proceedings of Machine Learning Research, pages 3040-3049, Long Beach, California, USA, 09-15 Jun 2019. PMLR. URL http://proceedings.mlr.press/v97/jaques19a.html.

Kate Jeffery, Robert Pollack, and Carlo Rovelli. On the statistical mechanics of life: Schrödinger revisited. Entropy, 21(12):1211, Dec 2019. ISSN 1099-4300. doi: 10.3390/e21121211. URL http://dx.doi.org/10.3390/e21121211.

Mateus Joffily and Giorgio Coricelli. Emotional valence and the free-energy principle. PLoS Computational Biology, 9(6):e1003094, 2013.

Daniel Kahneman and Gary Klein. Conditions for intuitive expertise: A failure to disagree. American Psychologist, 64(6):515-526, September 2009. 
Ivana Kajić, Tobias Schröder, Terrence C Stewart, and Paul Thagard. The semantic pointer theory of emotion: Integrating physiology, appraisal, and construction. Cognitive Systems Research, 58:35-53, 2019.

Emmanuel Kant. Groundwork of the Metaphysic of Morals. 1785.

Richard D. Lane. Neural correlates of conscious emotional experience. In Richard D. Lane and Lynn Nadel, editors, Cognitive Neuroscience of Emotion, chapter 15, pages 345-370. Oxford University Press, 2000.

Edward J. Lawler, Shane R. Thye, and Jeongkoo Yoon. Social Commitments in a Depersonalized World. Russell Sage Foundation, 2009.

Omar Lizardo and Michael Strand. Skills, toolkits, context and institutions: Clarifying the relationship between different approaches to cognition in cultural sociology. Poetics, 38:204$227,2010$.

G. Loewenstein and J.S. Lerner. The role of affect in decision making. In R.J. Davidson, K.R. Sherer, and H.H. Goldsmith, editors, Handbook of Affective Sciences, page 619-642. Oxford Univ. Press, 2003.

David J. C. MacKay. Information Theory, Inference, and Learning Algorithms. Cambridge University Press, 2003.

Neil J. MacKinnon and David R. Heise. Self, identity and social institutions. Palgrave and Macmillan, New York, NY, 2010.

Neil J. MacKinnon and Jesse Hoey. On the inextricability and complementarity of cognition and affect: A review and model. Under Review, 2020.

John Levi Martin and Alessandra Lembo. On the other side of values. American Journal of Sociology, 156(1):52-98, July 2020.

Karl Marx. Das Kapital. Kritik der politischen Ökonomie. Verlag von Otto Meisner, Germany, 1867.

George Herbert Mead. Mind, Self and Society. University of Chicago Press, 1934.

Hugo Mercier and Sperber. The Enigma of Reason. Harvard University Press, 2017.

Michael Moutoussis, Nelson J.Trujillo-Barreto, Wael El-Deredy, Raymond J. Dolan, and Karl J. Friston. A formal model of interpersonal inference. Frontiers in Human Neuroscience, 8(160), 2014.

Kevin P. Murphy. Probabilistic Machine Learning: An introduction. MIT Press, 2021. URL http://mlbayes.ai.

George Orwell. Animal Farm. Secker and Warburg, London, England, August 1945.

Charles E. Osgood, G. J. Suci, and Percy H. Tannenbaum. The Measurement of Meaning. University of Illinois Press, Urbana, 1957. 
Elinor Ostrom. Governing the Commons: The Evolution of Institutions for Collective Action. Canto Classics. Cambridge University Press, 2015. doi: 10.1017/CBO9781316423936.

Orlando Patterson. Making sense of culture. Annual Review of Sociology, 40:1-30, 2014.

Judea Pearl. Probabilistic Reasoning in Intelligent Systems: Networks of Plausible Inference. Morgan Kaufmann, San Mateo, CA, 1988.

David Pitcher and Leslie G. Ungerleider. Evidence for a third visual pathway specialized for social perception. Trends in Cognitive Sciences, $\mathrm{xx}(\mathrm{xx})$, Month 2020.

David Poole and Alan Mackworth. Artificial Intelligence: Foundations of Computational Agents. Cambridge University Press, 2010.

Pascal Poupart, Nikos Vlassis, and Jesse Hoey. An analytic solution to discrete bayesian reinforcement learning. In Proceedings of the International Conference on Machine Learning, Pittsburgh, USA, 2006.

William T. Powers. Behavior: The control of perception. Aldine publishing co., Chicago, 1973.

Martin L. Puterman. Markov Decision Processes: Discrete Stochastic Dynamic Programming. Wiley, New York, NY., 1994.

Joseph M. Quinn, Robert E. Freeland, Jesse Hoey, Kimberley B. Rogers, and Lynn Smith-Lovin. How the covid-19 pandemic changed the u.s. cultural meanings of essential and non-essential occupations. under review, 2021.

P.J. Richerson and R. Boyd. The evolution of human ultra-sociality. In I. Eibl-Eibesfeldt and F. K. Salter, editors, Indoctrinability, Ideology and Warfare: Evolutionary Perspectives, pages 71-95. Berghahn, New York, 1998.

Cecilia L. Ridgeway. Status: Why is it everywhere? Why does it matter? Russell Sage, 2019.

Jean-Jacques Rousseau. The Social Contract. 1762.

Stuart Russell and Peter Norvig. Artificial Intelligence: A Modern Approach. Prentice Hall, third edition, 2010.

Tobias Schröder, Jesse Hoey, and Kimberly B. Rogers. Modeling dynamic identities and uncertainty in social interactions: Bayesian affect control theory. American Sociological Review, 81 (4), 2016.

Dan Simon, Douglas M. Stenstrom, and Stephen J. Read. The coherence effect: Blending cold and hot cognitions. Journal of Personality and Social Psychology, 109(3):369-394, 2015.

Mario Luis Small, David J. Harding, and Michèle Lamont. Reconsidering culture and poverty. Annals of the American Academy of Political and Social Science, 629:6-27, May 2010.

Kevin B. Smith, John R. Alford, John R. Hibbing, Nicholas G. Martin, and Peter K. Hatemi. Intuitive ethics and political orientations: Testing moral foundations as a theory of political ideology. American Journal of Political Science, 61(2):424-437, April 2017. 
Rich Sutton and Andrew G. Barto. Reinforcement Learning: An Introduction. MIT Press, 2nd edition, 2018.

Robert B. Talisse. Overdoing Democracy: Why we must put politics in its place. Oxford University Press, 2019.

Paul Thagard. Hot thought: Mechanisms and applications of emotional cognition. MIT Press, 2006.

Johnathan H. Turner. The evolutionary biology and sociology of social order. In Edward J. Lawler, Shane R. Thye, and Jeongkoo Yoon, editors, Order on the Edge of Chaos, chapter 2, pages 18-42. Cambridge University Press, 2016.

Stephen Vaisey and Lauren Valentino. Culture and choice: Toward integrating cultural sociology with the judgment and decision-making sciences. Poetics, 68:131 - 143, 2018. ISSN 0304422X. doi: https://doi.org/10.1016/j.poetic.2018.03.002. URL http://www. sciencedirect. com/science/article/pii/S0304422X17301869.

Rufin VanRiullen and Ryota Kanai. Deep learning and the global workspace theory. under review, 2021.

Oriol Vinyals, Igor Babuschkin, Wojciech M Czarnecki, Michaël Mathieu, Andrew Dudzik, Junyoung Chung, David H Choi, Richard Powell, Timo Ewalds, Petko Georgiev, et al. Grandmaster level in Starcraft II using multi-agent reinforcement learning. Nature, 575(7782):350-354, 2019 .

John von Neumann and Oskar Morgenstern. Theory of Games and Economic Behavior. Princeton University Press, Princeton, NJ, 3 edition, 1953. 


\section{A Free energy}

I now derive the curves in Figure 3 from free energy principles. I will start with the free energy of the whole group of $\mathrm{N}$ agents considered as a single agent. I will denote the $i^{\text {th }}$ agent's parameters as $\theta_{i}$ and the parameters of the whole group as $\theta \equiv\left\{\theta_{1}, \ldots, \theta_{N}\right\}$. Thus, the task of the group at time $t$, given data as observations of emotional $(e)$ and other $(o)$ data $D=\{e, o\}_{t}=$ $\left\{e_{1}, e_{2}, \ldots, e_{t}, o_{1}, o_{2}, \ldots, o_{t}\right\}$ is to compute

$$
P(\theta \mid D, \mathcal{H})=\frac{P(D \mid \theta, \mathcal{H}) P(\theta \mid \mathcal{H})}{P(D \mid \mathcal{H})}
$$

where $\mathcal{H}$ is the hypothesis space (the modeling space as defined by the BayesAct model, for example). The graphical model for these agents is shown in Figure 9(a), shown with a single latent variable $\mathbf{Z}$ for the entire group. The difficult part here is the evaluation of $P(D \mid \mathcal{H})$ since it involves a summation over all values of $\theta$. Further, each of these terms involves sums over the hidden variables $\mathbf{Z}$.

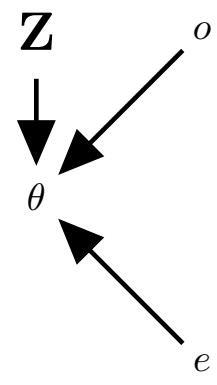

(a)

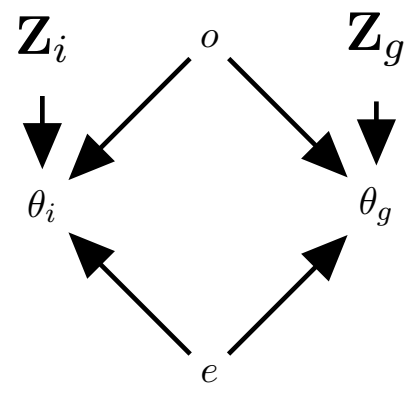

(b)

Figure 9: Simplified factor graph (Bayesian network) representing (a) a group with latent variables $\mathbf{Z}$; and (b) the group without agent $i$ with state $\mathbf{Z}_{g}$, and one representing agent $i$ with state $\mathbf{Z}_{i}$.

The free energy for this group model can be approximated with a variational function $Q(\theta)$, and the variational free energy, $\tilde{F}$, can be written as

$$
\tilde{F}=\int Q(\theta) \log \frac{Q(\theta)}{P(D \mid \theta)}
$$

When $Q$ and $\theta$ are chosen such that $\tilde{F}$ is minimised, then $\theta$ will be the best predictor function for this domain and agent combination (MacKay, 2003). The overall minimization process is normally handled by choosing $Q$ for some fixed (current best-guess) $\theta$, and then optimizing $\theta$ with respect to that "discovered" $\mathrm{Q}$, and repeating this process until convergence to a local minimum. The key idea is that by choosing the $\mathrm{Q}$ function appropriately, a minimization over model parameters is possible based on it, and this minimization will not leave our parameters any worse off as far as their relationship (fit) with the data goes. In many cases, Q may be determined from the data, but in some it may only be possible over some parameterised subset of the space of $Q$. For example, $Q$ can be factored into parts corresponding to each parameter, 
and then each such factored $Q$ can be minimised analytically one at a time, while keeping the others constant. The variational $Q$ is in fact a "functional" which means a family of functions. The choice of this functional may be important, but typical solutions are found empirically or using very simple functionals. The method works by first "fitting" the functional $Q$ to the probability distribution being optimised at some "guess" for $\theta$. and then optimizing $\theta$ over the function so defined. This process is repeated until convergence and may be superior at finding the global optimum

In order to move beyond the group to each individual agent, I will split the group into two parts. One singleton set contains the $i^{t h}$ agent, with parameters $\theta_{i}$ and latent variables $\mathbf{Z}_{i}$, and the other set contains $N-1$ agents $\{1, \ldots, N\} \backslash i$, with parameters $\theta_{g}$ and latent variables $\mathbf{Z}_{g}$. I will consider this second set of agents as a single agent as I did above for the whole group. The role of this single "group" agent is, in fact, taken by a single individual from the group at any one time, but the statistics of interaction of the agent in question with the whole group is what matters, as I will show. At this point, I encourage you to think of this as a dyadic interaction, but it can also be viewed as an agent-group interaction, or even a group-group interaction. It is known that group identity (connotative) emerges as associated with specific group labels (denotative), and that these labels, if applied to groups, imply an a priori bias towards that connotative identity in individuals (Ridgeway, 2019, p.133,p.148).

In this restricted case, each agent is then trying to optimise its own posterior density (including over its own action space),

$$
P\left(\theta_{i} \mid D, \mathcal{H}_{i}\right)=\frac{P\left(D \mid \theta_{i}, \mathcal{H}_{i}\right) P\left(\theta_{i} \mid \mathcal{H}_{i}\right)}{P\left(D \mid \mathcal{H}_{i}\right)}
$$

and the network model looks as in Figure 9(b).

I will now assume that the variational distribution for the group, $Q$, for any agent $i$, can be factored into a piece for the agent, $Q_{i}\left(\theta_{i}\right)$ and a piece for the group, $Q_{g}\left(\theta_{g}\right)$, such that $Q(\theta)=Q_{i}\left(\theta_{i}\right) Q_{g}\left(\theta_{g}\right)$. As explained above, a variational solution to this model will normally require some kind of iterative updating scheme like the expectation-maximization algorithm, which operates by optimising one parameter at a time, while holding the others fixed. This kind of iterative solution in achieved by factorizing the group into individuals optimizing their own $Q$ functions, based on everyone else's $Q$ functions, assuming they are fixed. For the entire group I am considering, I am assuming that each agent can separately and independently minimise its own variational free energy.

What this implies is that the quantity each agent in a group of agents will be optimizing is

$$
\tilde{F}=\int Q_{i}\left(\theta_{i}\right) Q_{g}\left(\theta_{g}\right) \log \frac{Q_{i}\left(\theta_{i}\right) Q_{g}\left(\theta_{g}\right)}{P_{i}\left(D \mid \theta_{i}\right) P_{g}\left(D \mid \theta_{g}\right)}
$$

Consider $D$, the total data generated by the agent+group. I will break this into three nonoverlapping sets, $D \equiv\left\{D_{o}, D_{i}, D_{g}\right\}$ where $D_{i}$ is the data generated during the interaction by the agent, $i$, while $D_{g}$ is the data generated by the group, $g$, and $D_{o}$ is the data generated by both simultaneously. The goal of the optimization is to get $D_{i}$ to be interpretable by the group, to get $D_{g}$ to be interpretable by the agent, and to get $D_{o}$ to be interpretable by both. Both agent and group will be updating their models, $\theta_{i}$ and $\theta_{g}$, respectively, during the interaction. I will therefore simplify by assuming that each agent generates "its" data, then observes $D_{i}$ and 
$D_{g}$, and then generates the shared data $D_{0}$. Then I can factor

$$
\begin{aligned}
& P_{i}\left(D \mid \theta_{i}\right) \quad=\int_{\theta_{i}^{\prime}} P_{i}\left(D_{o}, D_{i}, D_{g}, \theta_{i}^{\prime} \mid \theta_{i}\right) \\
& =\int_{\theta_{i}^{\prime}} P_{i}\left(D_{o} \mid D_{i}, D_{g}, \theta_{i}^{\prime}, \theta_{i}\right) P_{i}\left(\theta_{i}^{\prime} \mid D_{g}, D_{i}, \theta_{i}\right) P_{i}\left(D_{i} \mid D_{g}, \theta_{i}\right) P\left(D_{g} \mid \theta_{i}\right) \\
& =\int_{\theta_{i}^{\prime}} P_{i}\left(D_{o} \mid \theta_{i}^{\prime}\right) P_{i}\left(\theta_{i}^{\prime} \mid D_{g}, D_{i}, \theta_{i}\right) P_{i}\left(D_{i} \mid \theta_{i}\right) P_{i}\left(D_{g} \mid \theta_{i}\right) \\
& P_{g}\left(D \mid \theta_{g}\right) \quad=\int_{\theta_{g}^{\prime}} P_{g}\left(D_{o}, D_{i}, D_{g}, \theta_{g}^{\prime} \mid \theta_{g}\right) \\
& =\int_{\theta_{g}^{\prime}} P_{g}\left(D_{o} \mid \theta_{g}^{\prime}\right) P_{g}\left(\theta_{g}^{\prime} \mid D_{i}, D_{g}, \theta_{g}\right) P_{g}\left(D_{g} \mid \theta_{g}\right) P_{g}\left(D_{i} \mid \theta_{g}\right)
\end{aligned}
$$

where I have assumed that $D_{o}$ is generated from updated models in agent $\theta_{i}^{\prime}$ and group $\theta_{g}^{\prime}$ after seeing $D_{i}$ and $D_{g}$. Further, I've assumed each agent computes its own $P(D \mid \theta)$ without considering the other's data. That is, $P_{i}\left(D_{i} \mid D_{g}, \theta_{i}\right)=P_{i}\left(D_{i} \mid \theta_{i}\right)$ and $P_{g}\left(D_{i} \mid D_{g}, \theta_{g}\right)=P_{g}\left(D_{i} \mid \theta_{g}\right)$. Putting Equations 5 and 6 back into Equation 4 , and rearranging terms, I obtain:

$$
\begin{aligned}
\tilde{F}=\int Q_{i}\left(\theta_{i}\right) & Q_{g}\left(\theta_{g}\right) \log \frac{Q_{i}\left(\theta_{i}\right)}{P_{i}\left(D_{i} \mid \theta_{i}\right) \int_{\theta_{i}^{\prime}} P_{i}\left(D_{o} \mid \theta_{i}^{\prime}\right) P_{i}\left(\theta_{i}^{\prime} \mid D_{g}, D_{i}, \theta_{i}\right)} \\
& +\int Q_{i}\left(\theta_{i}\right) Q_{g}\left(\theta_{g}\right) \log \frac{Q_{g}\left(\theta_{g}\right)}{P_{i}\left(D_{g} \mid \theta_{i}\right) P_{g}\left(D_{i} \mid \theta_{g}\right) P_{g}\left(D_{g} \mid \theta_{g}\right) \int_{\theta_{g}^{\prime}} P_{g}\left(D_{o} \mid \theta_{g}^{\prime}\right) P_{g}\left(\theta_{g}^{\prime} \mid D_{i}, D_{g}, \theta_{g}\right)}
\end{aligned}
$$

Now I will evaluate this free energy at a fixed point where $\theta_{g}^{\prime}=\theta_{g}$ and $\theta_{i}^{\prime}=\theta_{i}$, and in the particular case where $D_{o}=\varnothing$, ergo, the group and individual are at equilibrium. This means neither agent nor group changes parameters based on the other's data. However, at equilibrium, where I am computing this equilibrium, it allows us to compute the integrals in closed form, and thus is a fixed point solution. Thus, in Equation $7 \mathrm{I}$ can set $Q_{g}=P_{g}\left(D_{g} \mid \theta_{g}\right)$ and set the integrals over $\theta_{i}^{\prime}$ and $\theta_{g}^{\prime}$ in the denominators to identity (since one term picks out $\theta^{\prime}=\theta$ and the other is $P\left(D_{o}=\varnothing \mid \theta\right)=1$ they pick out the equilibrium point, which is the starting point).

With these assumptions in hand, I can rewrite Equation 7 as:

$$
\begin{aligned}
\tilde{F}=\int_{\theta_{g}} Q_{g}\left(\theta_{g}\right) & \int_{\theta_{i}} Q_{i}\left(\theta_{i}\right) \log \frac{Q_{i}\left(\theta_{i}\right)}{P_{i}\left(D_{i} \mid \theta_{i}\right)} \\
& -\int_{\theta_{i}} Q_{i}\left(\theta_{i}\right) \int_{\theta_{g}} Q_{g}\left(\theta_{g}\right) \log P_{i}\left(D_{g} \mid \theta_{i}\right) P_{g}\left(D_{i} \mid \theta_{g}\right)
\end{aligned}
$$

The first term is the usual free energy for the agent, averaged over models of the group. However, assuming the group is stationary, then the free energy of the agent then resolves to its own free energy, which can be computed. The second term is the joint probability that agent $i$ will be able to generate data $D_{i}$ that is interpretable by the group, and that the group will be able to generate data $D_{g}$ that is interpretable by the agent. This is taken in expectation over both models of agent and group parameters, $Q_{i}$ and $Q_{g}$.

Note the symmetry in Equation 8 , in which the dispersion of $\theta_{i}$ can be large if the dispersion of $\theta_{g}$ is small, and vice-versa, but both can't be large or small at the same time. To return to our flashlights, if the target is large (the dispersion of $\theta_{g}$ is large), then the beam must be small (the dispersion of $\theta_{i}$ must be small. In fact, this symmetry is quite curious because it states that individuals operating in the first regime will be well suited to interact with individuals 
operating in the second. That is, although they are doing things differently, they in fact are complementary. In this case, the targets themselves are also the inverse beams coming from the group. There is a tradeoff between the width of these beams, with insufficient density if the two are large, difficulty finding the other if the two are small, leaving the agents to find tradeoffs in the middle. The exact location of this tradeoff is then something that must be negotiated, and may easily involve stereotypes of status. It also determines the sets $D_{o}, D_{i}, D_{g}$ defined above, since e.g. if the dispersion of $\theta_{g}$ is small, most of the data will be generated by the group, so $D_{o}=D_{g}$, and the group will be considered "high status", while the individual "low status." If the dispersion of $\theta_{g}$ is large, the dispersion of $\theta_{i}$ is small, and so $D_{o}=D_{i}$ and the individual is considered "high status", while the group "low status."

Focusing on the second term in Equation 8 only, I can expand out the logarithm and get two terms

$$
-\int_{\theta_{i}} Q_{i}\left(\theta_{i}\right) \int_{\theta_{g}} Q_{g}\left(\theta_{g}\right) \log P_{i}\left(D_{g} \mid \theta_{i}\right)-\int_{\theta_{g}} Q_{g}\left(\theta_{g}\right) \int_{\theta_{i}} Q_{i}\left(\theta_{i}\right) \log P_{g}\left(D_{i} \mid \theta_{g}\right),
$$

which I can optimise separately. Holding the agent fixed at $\theta_{i}^{*}$ and optimizing $\theta_{g}$ in the first, and holding the group fixed at $\theta_{g}^{*}$ and optimising $\theta_{i}$ in the second. Then, this is

$$
-\int_{\theta_{g}} Q_{g}\left(\theta_{g}\right) \log P_{i}\left(D_{g} \mid \theta_{i}^{*}\right)-\int_{\theta_{i}} Q_{i}\left(\theta_{i}\right) \log P_{g}\left(D_{i} \mid \theta_{g}^{*}\right)
$$

Now, $D_{g}=\left\{d_{g 0}, d_{g 1}, \ldots, d_{g N}\right\}$ and $D_{i}=\left\{d_{i 0}, d_{i 1}, \ldots, d_{i N}\right\}$, which means, assuming all the data are independently and identically distributed given each model, we can write this as

$$
-\sum_{j}\left[\int_{\theta_{g}} Q_{g}\left(\theta_{g}\right) \log P_{i}\left(D_{g j} \mid \theta_{i}^{*}\right)+\int_{\theta_{i}} Q_{i}\left(\theta_{i}\right) \log P_{g}\left(D_{i j} \mid \theta_{g}^{*}\right)\right],
$$

I will assume that $Q_{g}$ is a "hat" function which has constant probability over $\left[\mu_{g}^{*}-\sigma_{g}^{*}, \mu_{g}^{*}+\sigma_{g}^{*}\right]$ (so $\left.\theta_{g}^{*}=\left\{\mu_{g}^{*}, \sigma_{g}^{*}\right\}\right)$. Similarly for $Q_{i}$ : replace all $g$ subscripts with $i$. Next, I assume that $P_{i}$ and $P_{g}$ are normal distributions with parameters $\theta_{i}=\left\{\mu_{i}, \sigma_{i}\right\}$ and $\theta_{g}=\left\{\mu_{g}, \sigma_{g}\right\}$, and then the integrals can be done analytically to yield, for each data point, a contribution to the overall free energy of (note the extra negative sign that came from the $\log$ (Normal) distributions)

$$
\frac{\left(\mu_{g}+\sigma_{g}-\mu_{i}^{*}\right)^{3}}{3 \sigma_{i}^{* 2}}-\frac{\left(\mu_{g}-\sigma_{g}-\mu_{i}^{*}\right)^{3}}{3 \sigma_{i}^{* 2}}+\frac{\left(\mu_{i}+\sigma_{i}-\mu_{g}^{*}\right)^{3}}{3 \sigma_{g}^{* 2}}-\frac{\left(\mu_{i}-\sigma_{i}-\mu_{g}^{*}\right)^{3}}{3 \sigma_{g}^{* 2}} .
$$

Now, to plot this, I assume we are at equilibrium, which I set at zero, so that $\mu_{g}^{*}=\mu_{i}^{*}=0$ and $\sigma_{i}^{*}=\sigma_{g}^{*}=1$ and I obtain two terms as follows

$$
\left[\frac{\left(\mu_{g}+\sigma_{g}\right)^{3}}{3 \sigma_{i}^{* 2}}-\frac{\left(\mu_{g}-\sigma_{g}\right)^{3}}{3 \sigma_{i}^{* 2}}\right]+\left[\frac{\left(\mu_{i}+\sigma_{i}\right)^{3}}{3}-\frac{\left(\mu_{i}-\sigma_{i}\right)^{3}}{3}\right] .
$$

The first is the negative log probability (free energy) that the group will align with the agent, which will be lower (more probable, lower free energy) if the agent is more "flexible" (can show a face the group will like, blue line in Figure 3). The second is the negative log probability the agent will align with the group, which will be lower if the agent is more precisely defined (i.e. 
more "findable," red line in Figure 31. Although in this case it is simply because I assumed we were at equilibrium, but in general will be true because the group can more easily "place" the newcomer.

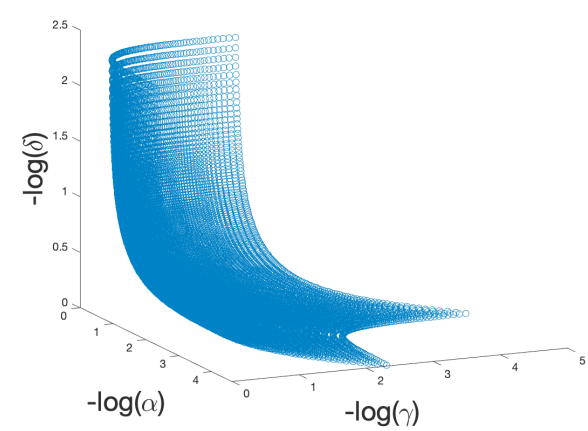

(a)

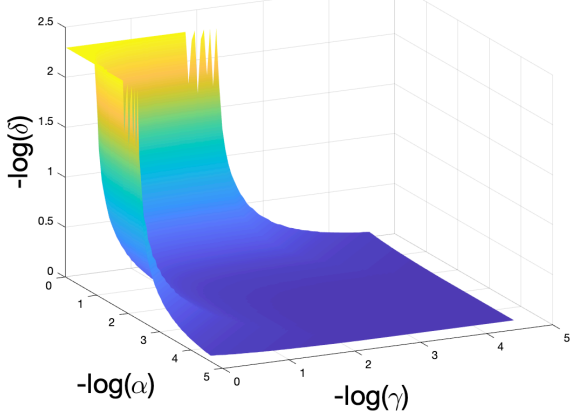

(c)

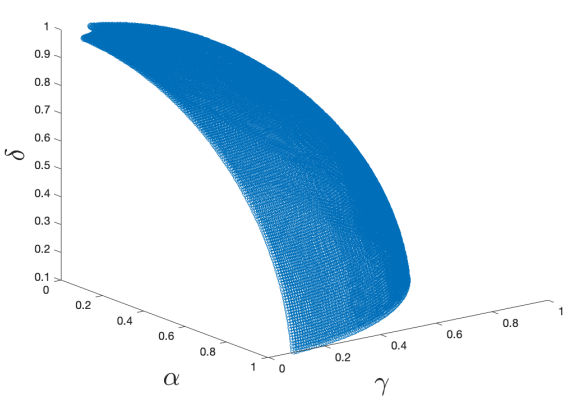

(b)

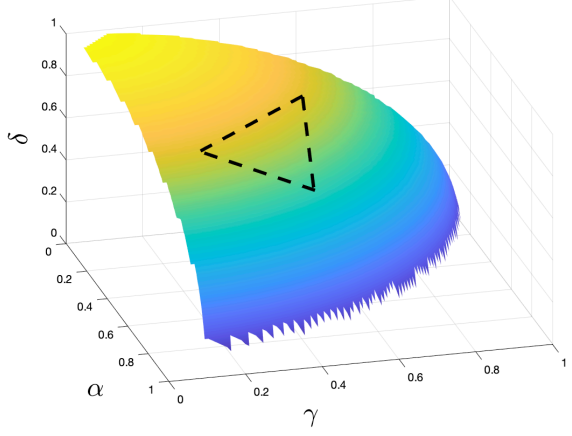

(d)

Figure 10: Views of the minimum free energy points along each ray from the origin. (a) and (c): Axes are $-\log ($ parameters), but correspond in scale to coherence $(-\log (\hat{\alpha}))$, dependence $(-\log (\gamma))$, and validity $(-\log (\delta))$. I plotted the negative logarithm of each parameter, and colored the surface in Figure 10(c), for visibility only. (b) and (d): Axes are the raw variance parameters (so larger is more dispersed, more freedom). The triangle in Figure (d) is a stylistic representation of a potential range of workable settings, lying in a simplex-like shape.

Now I remind you that the first term in Equation 10 in fact represents the entire group of $N-1$ individuals. Therefore, by weighting the two terms equally (black line in Figure 3), I have made an implicit assumption that the group is fully connected, so that there are $N-1$ terms like the first in Equation 10, and $N-1$ terms like the second, that is, the individual interacts with everyone. This is not likely to be the case, however. What is more likely is that the newcomer interacts with only a dozen colleagues and managers, so his influence on the group is small. If we approximate this linearly and weight the first term in Equation 10 by $N$, then the dashed curves in Figure 3 result. The optimal free energy has shifted outwards, and more individual flexibility is called for in order to integrate the individual into the group.

Nevertheless, the individual is going to have to change more than the group, as the weight of the population is in their favor (he is outnumbered). However, if the individual's parameters 
are substantially mis-aligned in general with the group's but aligned with some sub-group's parameters (setting of coherence, dependence and validity/ideology/moral matrix), then if the social network is constructed in such a way that this individual is mostly interacting with the sub-group, then these mis-aligned values may be strengthened within the sub-group. Should the group become large enough, or socially organised enough, their skepticism may be able "to offer a challenge to the upholders of the 'official' tradition" (Berger and Luckmann, 1967, p.121). This challenge may be handled by merger into the main institution (internalization by the sub-group of the primary group), which then enriches and differentiates this tradition, or by segregation of the skeptics, a process of objectivation that possibly includes dehumanization (change of their agreed upon assigned identity). Finally, the sub-group may gain sufficient strength to trigger a revolution, in which case the existing traditions are thrown away and replaced with the new ideology, and the sub-group externalises to the group, society is produced by this sub-group, who define the new reality (Berger and Luckmann, 1967).

Equation 10 is the free energy for a one dimensional parameter space, under certain assumptions. In the three dimensional space shown in Figure 6, we can imagine this free energy curve, as shown in Figure 3, varies along any ray emanating from the origin, and that the minimum point defines the surface of the "simplex," which is therefore revealed to be more of a "dome" shape (assuming radial symmetry). I therefore plot the simplex by seeking the minimum free energy along each ray from the origin. Plotting this as a function of $-\log \theta \propto \theta^{-1}$ ) yields Figure 10(a), with an interpolated, smoothed version in (c). Figure $3(\mathrm{~b})$ and (d) are the same plotting $\theta$ directly. One can see it is not, in fact, a simplex. Nevertheless, recall that a social system becomes increasingly difficult to arrange as you move out along any dimension of freedom, and thus the actual range of operation of these parameters is likely to be relatively small, centered around a region in center of the minimum free energy manifold, shown stylistically as a triangle in Figure 3(d). Further, the simplex gives us a convenient way to discuss the manifold shown in Figure 10, so long as we remember that in practice it has this particular scaling. Note that the minimum free energy goes to $\infty$ as any parameter drops to zero, as Equation 10 blows up. It is therefore more difficult to plot the simplex as a function of the inverses of the parameters (Figure 3(a) and (b)).

There are numerous assumptions and shortcuts in the above analysis, but my objective was to derive a first approximation to this function. The assumption that group and agent are fixed are quite restrictive, and the analysis above simplifies the simultaneous change of agent to group and from group to agent (which change may be modulated by the "gravity" of the group) by using the symmetry of the problem. This simplification allows me to hold one agent fixed and modulate the other (or hold the group fixed and modulate the agent). Nevertheless, any more complex and reciprocal change would be characterised by the same equations, except with perhaps a coordinate change. Thus, I have proceeded with loss of generality only in the assumptions made ( $\operatorname{such}$ as $D_{o}=\varnothing$ ), but relaxing these assumptions would generate multiple interesting avenues for future work.

Generality is also reduced by the fact that I left out external forces altogether. However, such forces could be added to the equations above as an additional element that could be modeled, and would share responsibility for D (along with the agent and group). Adding such elements may skew the overall structures shown in Figure 10, but will not change the core ideas I am presenting. This does, however, remain for a topic of future research. 


\section{B Political belief data - significance}

First, I consider significance of the difference in variance across all occupations, and therefore assume that the two datasets are independent samples of a single measure of variance. One-way f-tests (which are simply the ratio of variances) across the 85 occupations gave coefficients as shown in Table 1, and these show some degree of significance in the difference between Demorats and Republicans (with p-values of $0.02,10^{-3}, 10^{-8}$ for e,p,a respectively. However, f-tests may be invalid for unequal variances (which is what we have) so the Kruskal-Wallis test is used instead, and generates p-values shown in Table 1. Mann-Whitney tests were also performed as shown. The Kruskal-Wallis test shows a significant increase in variance from republican to democrat for all dimensions with power being having the smallest significance $p<0.05$, and activity having the biggest $p<10^{-7}$. Democrats also showed significantly higher variance over independents in potency $(p<0.05)$ and activity $\left(p<10^{-6}\right)$, but independents showed significantly higher variance than republicans in evaluation $(p<0.01)$ only.

Second, I consider paired significance, so that each occupation is treated separately and compared across political belief lines. In this case, the Wicoxon signed rank test ${ }^{14}$ shows significance for all pairs except democrats and independents on evaluation, and republicans and independents on activity. Otherwise, the most significant differences are found for the differences between democrats and republicans on activity and potency. Strong differences are also found betwen democrats and republicans on activity ratings.

This data provides evidence that the variances are, on the average, significantly higher for people who rate themselves as more strongly republican, and lower for those who rate themselves more strongly democrat. This is in agreement with the overall statement of this paper.

One other thing I will note is that, when looking at only the essential occupations, the variance increase is about the same (Wilcoxon $p=0.01,0.02$, and $10^{-5}$ for e,p, and a, respectively). However, when looking at non-essential, the variance stays about the same (Wilcoxon is only significant for activity: $\mathrm{p}=0.14,0.22,0.006$ for $\mathrm{e}, \mathrm{p}, \mathrm{a})$. It may be the case that democrats had more sensitivity to these labels than republicans. The trend of an increase in variance are still visible, see Figure 11 .

\footnotetext{
${ }^{14}$ The Wilcoxon takes the difference between sums of ranks in absolute difference. That is, it first discards all identical data, then ranks all pairwise absolute differences (in variance in this case). Suppose we are comparing $\hat{\gamma}_{R}$ esimated for republicans (participants who said they were republican) and $\hat{\gamma}_{D}$ estimated for democrats. Then the test computes the following as a statistic: $\left(\sum\right.$ ranks where $\left.\hat{\gamma}_{D}>\hat{\gamma}_{R}\right)-\left(\sum\right.$ ranks where $\left.\hat{\gamma}_{R}>\hat{\gamma}_{D}\right)$.
} 
evaluation

\begin{tabular}{llccc} 
& & Democrat & Independent & Republican \\
\hline Wilcoxon & Democrat & - & $1689 / 0.54$ & $1254 / 0.01^{*}$ \\
& Independent & & - & $1132 / 0.002^{* *}$ \\
\hline Kruskal- & Democrat & - & $0.06 / 0.81$ & $9.11 / 0.003^{* *}$ \\
Wallis & Independent & & - & $8.86 / 0.003^{* *}$ \\
\hline Mann- & Democrat & - & $3533 / 0.40$ & $2644 / 0.001$ \\
Whitney-U & Independent & & - & $2658 / 0.002^{* *}$ \\
\hline f-test & Democrat & - & $0.03 / 0.85$ & $5.1 / 0.02^{*}$ \\
& Independent & & - & $4.5 / 0.04^{*}$ \\
\hline \hline
\end{tabular}

\begin{tabular}{llccc}
\hline \hline \multicolumn{5}{c}{ potency } \\
& & Democrat & Independent & Republican \\
\hline Wilcoxon & Democrat & - & $1242 / 0.01^{*}$ & $989 / 0.0002^{* * *}$ \\
& Independent & & - & $1340 / 0.03^{*}$ \\
\hline Kruskal- & Democrat & - & $5.6 / 0.02^{*}$ & $6.2 / 0.01^{*}$ \\
Wallis & Independent & & - & $.79 / 0.37$ \\
\hline Mann- & Democrat & - & $2850 / 0.009^{* *}$ & $2814 / 0.007^{* *}$ \\
Whitney U & Independent & & - & $3326 / 0.19$ \\
\hline f-test & Democrat & - & $5.3 / 0.02^{*}$ & $11.4 / 0.0009^{* * *}$ \\
& Independent & & - & $2.2 / 0.14$ \\
\hline \hline & & activity & & \\
& & Democrat & Independent & Republican \\
\hline Wilcoxon & Democrat & - & $626 / 10^{-7 * * *}$ & $448 / 10^{-9 * * *}$ \\
& Independent & & - & $1522 / 0.18$ \\
\hline Kruskal- & Democrat & - & $23 / 10^{-6 * * *}$ & $26 / 10^{-7 * * *}$ \\
Wallis & Independent & & - & $.54 / 0.46$ \\
\hline Mann- & Democrat & - & $2073 / 10^{-6 * * *}$ & $1978 / 10^{-7 * * *}$ \\
Whitney U & Independent & & - & $3375 / 0.23$ \\
\hline f-test & Democrat & - & $22 / 10^{-6 * * *}$ & $32 / 10^{-7 * * *}$ \\
& Independent & - & $1.4 / 0.23$ & \\
\hline
\end{tabular}

Table 1: Wicoxon signed rank, Kruskal-Wallis H-test, Mann-Whitney U and f-test statistics/pvalues for different pairs of political affiliations. The Democratic/Republican split can be clearly seen with p-values of $0.01,0.0002$ and $10^{-9}$ for $e, p$, and $a$, respectively. $p<0.05^{*} ; p<0.01 * *$; $p<0.001^{* * *}$. 


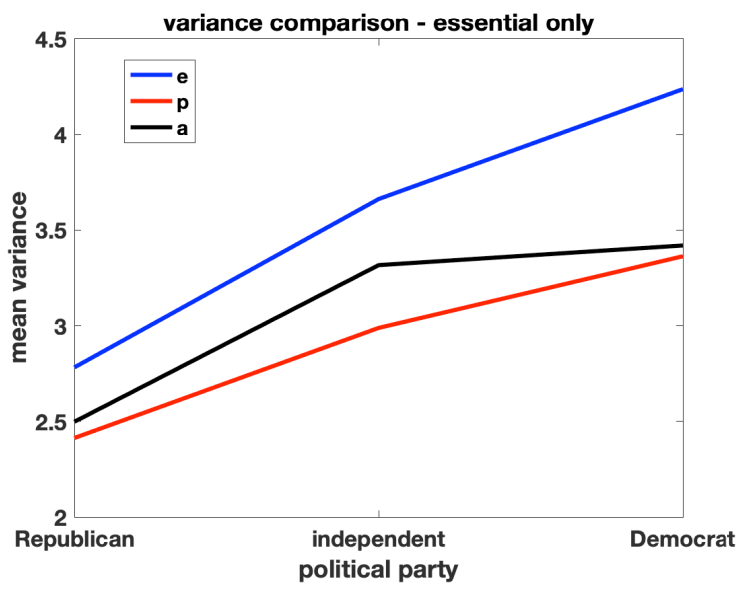

(a)

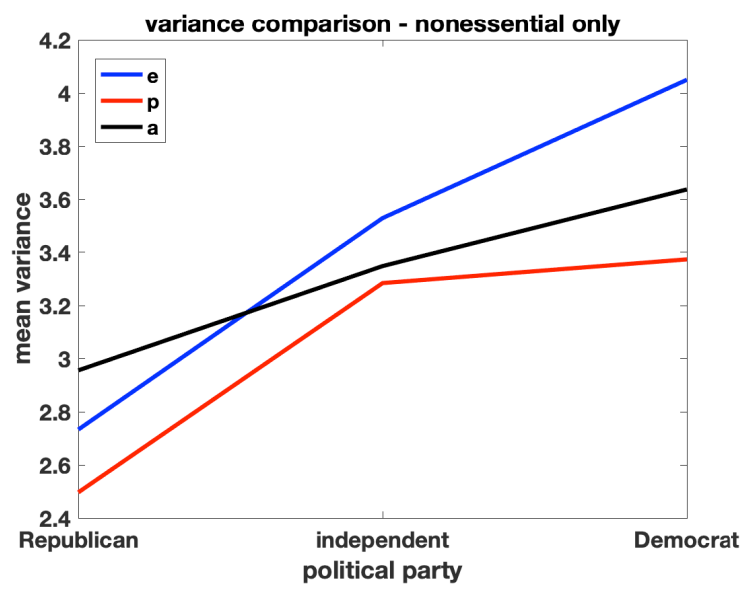

(b)

Figure 11: Data showing changes in variance in sentiments as a function of political orientation for essential and (b) non-essential. Compare to Figure 8 for all occupations. One can see a clear trend that correlates variance with left-leaning political beliefs. 